\title{
Determination of Concentration Profile for Flowing Solid Particles in Pipeline Using Electric Charge Tomography System
}

\author{
Iliya Tizhe Thuku, Mohd Fua'ad Rahmat, Norhaliza Abdul Wahab, and Teimor Tajdari \\ Control and Mechatronics Engineering Department, Faculty of Electrical Engineering, Universiti Teknologi Malaysia, \\ 81310 Skudai, Johor Bahru, Malaysia \\ Correspondence should be addressed to Mohd Fuảad Rahmat; fuaad@fke.utm.my
}

Received 7 August 2013; Revised 4 November 2013; Accepted 10 December 2013; Published 18 February 2014

Academic Editor: Alexei Mailybaev

Copyright (C) 2014 Iliya Tizhe Thuku et al. This is an open access article distributed under the Creative Commons Attribution License, which permits unrestricted use, distribution, and reproduction in any medium, provided the original work is properly cited.

\begin{abstract}
Tomography aims to present an image of a cross-sectional distribution of materials in some regions of interest such as crosssection of a pipeline or process vessel. This paper presents the concentration profiles of solid particles across a conveying pipeline obtained using tomographic imaging. In the paper, 16 electrodynamic sensors were installed around the circumference of a pipeline to capture electric charges carried by the particles moving through the pipeline under gravity. The Cartesian coordinate system used to derive the system equation gave an accurate charge distribution while the meshing technique of the finite element method applied miniaturized the pixel sizes within the sensing zone. The problem of unstable matrix and weak signal response around the center of the pipe cross-section, normally associated with the electric charge tomography system, was addressed using matrix compression through transposition and filtering. The pro rata distribution method mostly applied in the financial accounting analysis was used in the final stage. An algorithm for realization of the concepts was developed using MATLAB. The qualities of the resulting images for four different flow regimes provide good quality images representing the distribution of the particles across the pipeline crosssection.
\end{abstract}

\section{Introduction}

Electrical tomography technology involves taking measurement of electrical quantities of materials moving through a process vessel or conveying pipeline. The measurements are taken at the periphery of the vessel or pipeline using capacitance, electrostatic, impedance, electromagnetic, and so forth phenomena. With an appropriate algorithm, information on the electrical properties of the materials across a cross-section of the pipeline or process vessel can be obtained, making it appropriate for diverse industrial operation requirements [1] such as particles flow monitoring. The tomography concept has been employed in many areas of physical sciences and engineering in which measurement of parameters distributions or concentration profile of process materials in a region of interest is made [2].

Numerous sensing methods exist for process tomography in which sensor choice depends on purpose and conditions of measurement. According to Machida and Scarlett [3], most requirements that influence sensor choice include spatial resolution, physical properties of the material, measurement time, and environmental factors, such as pressure and temperature. The electrodynamic sensors are mostly employed in electric charge technique in the measurement of gas-solid flow parameters, because it consists of simple and cheap electrodes that do not require an external source of power and their performance is not affected by the environmental factors [4]. Pipeline conveyance is a technique used in many industries to convey solid materials from one point to the other [5]. The technique is mostly utilized in coal power plants, cement industries, food processing, and pharmaceutical industries to improve production efficiencies [6]. However, the electrodynamic sensors are used to detect charges carried by the moving particles, such that, when the charged particles travel through the sensing area where the sensors are installed, an unpredictable electrostatic field is created by the charges on the particles, leading to induced charges on the sensors. Consequently, the flow parameters, such as velocity, mass flow rate, particle size, and concentration profile of the moving particles, can be obtained $[7,8]$. In 


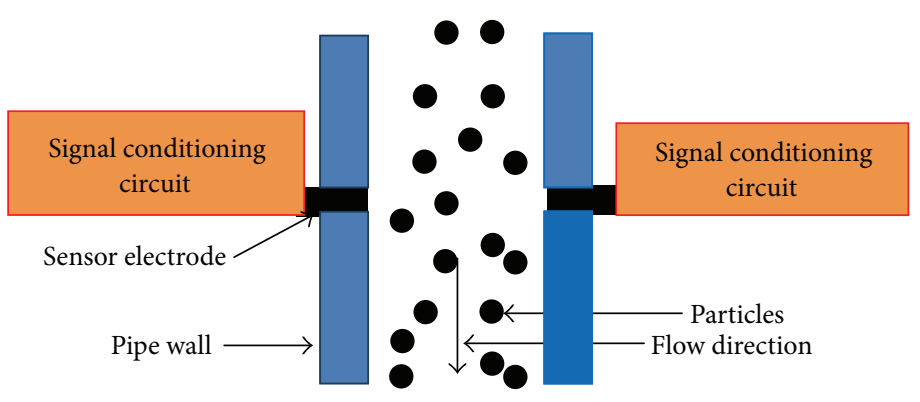

FIGURE 1: Cross-section of pipe and electrodynamic sensor installed for concentration measurement.

the determination of the concentration profile of particles, tomographic technology is usually applied. In tomographic image development, one of the most important requirements is a sensitivity map which is the presentation of the ability of sensors installed around a sensing zone to sense parameters of interest at any point in the zone. The main problem of tomographic imaging of multiphase flow is the quality of resultant charge distribution within the sensing zone [9]. Several efforts have been made to improve the quality of the image as reported in [10,11] with no specific solution to the problem of particle distribution within the sensing zone. Concentration profile or the tomographic imaging is one of the inverse problems characterized by ill-posed and ill-conditioned phenomenon, where small changes in the data cause arbitrarily large changes in the solution making it unstable [8].

The purpose of this paper is to present an efficient method that produces a stable system by which concentration profile or tomography image of solid particles moving through a pipeline can be achieved. The finite-element method (FEM) is a powerful tool that has not been much exploited in electric charge tomography (EChT) imaging system [12]. In the paper, 16 electrodynamic sensors were used, and forward model system equation was developed using Cartesian coordinate techniques [13]. In the development of the system's model equation, a two-dimensional induced charge model on each of the 16 electrostatic sensors installed around a conveying pipeline, using elements' techniques of FEM, is made. The system equation development also involved structural meshing of the sensing zone (pipeline cross-section) into triangular mesh elements, using MATLAB program. On each of the meshed elements, electrostatic laws were applied to develop the system equation of the particle charge distribution within the sensing zone.

The developed system equation was used to generate the sensitivity matrix which was filtered and normalized. The sensitivity matrix was used to generate the image matrix through application of a newly introduced tomography image reconstruction method, which we called pro rata distribution (PRD) technique. The PRD is a well-established concept in financial accounting analysis $[14,15]$ and has been effectively applied to concentration profiling in this paper. Experiments were carried out on plastic bead particles using a standard gravity flow electric charge tomography test rig; the introduced concepts of FEM and PRD gave stable results and good cross-sectional distribution of the plastic bead particles through the pipeline for four different artificially created flow profiles or regimes.

\section{The Forward Modelling of the Electric Charge Tomography System for the Concentration Profile}

2.1. The Electrodynamic Sensor. As mentioned earlier, electrodynamic sensors play the most important part in the electrical charge tomography system. During the particles movement through the pipeline, there are interactions among the flowing particles, conveying pipeline wall and the transporting gas, which creates electrostatic charges on the particles [4]. The charges carried by the particles are detected by electrodynamic sensors installed around the sensing zone of the conveying pipeline and are converted to voltages. The captured voltages are used for the concentration profiling through the application of tomography imaging algorithm. The sensor consists of a plain metal rod, called the electrode, which protrudes through the conveying pipeline wall and flushed on the inner surface. The sensor electrode is supported by signal conditioning circuits which process the captured charges into a voltage signal used for the concentration profiling system. The magnitude of the charges depends on many factors such as physical properties like shapes, sizes, density, conductivity, permittivity, chemical composition of the materials, and humidity [16]. Other major factors contributing to the magnitude of the charges are pipeline wall roughness, diameter, the pipe length traversed by the moving particles, particles velocity, and concentration [17]. A diagram showing cross-section of two electrodynamic sensors installed in the pipe is shown in Figure 1.

The charge conditioning circuit is designed to process the charges induced by the sensor electrodes due to the charges carried by the moving solid particles. Figure 2 shows a block diagram of complete circuit of the electrodynamics sensor. It consists of several major parts of which are sensor electrode, amplifier, precision rectifier, low-pass filter, and outputs.

In Figure 2, output 1 is an alternating component of the charge signal, normally used for velocity measurement. Output 2 is rectified voltage, which can be used for spatial filtering tests. The last output 3 is an averaged DC voltage, which is the signal of interest normally used for concentration measurement and flow regimes identification 


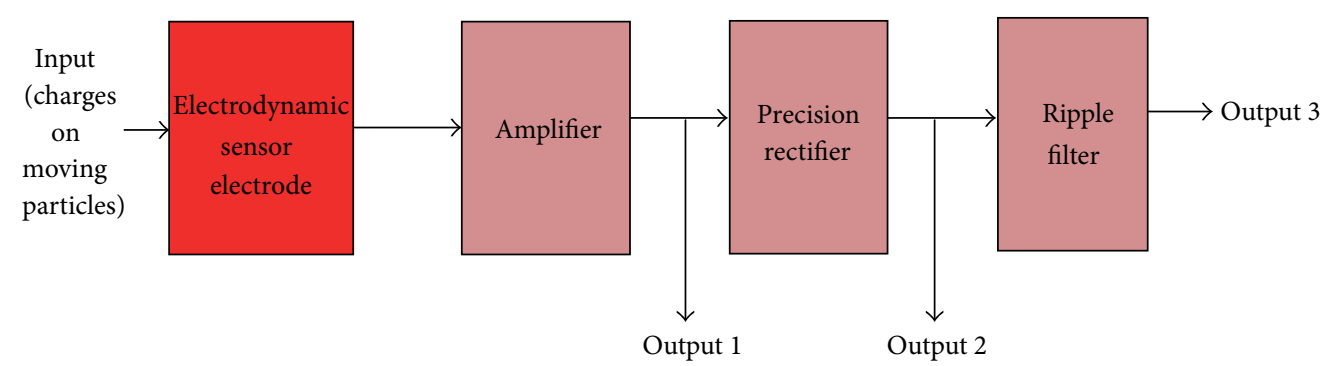

FIGURE 2: A block diagram of an electrodynamics sensor circuit.

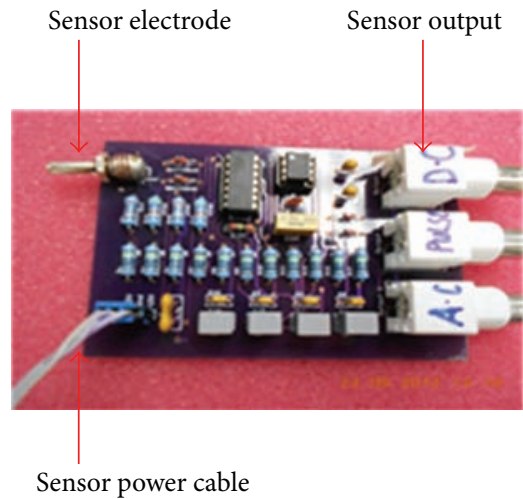

(a)

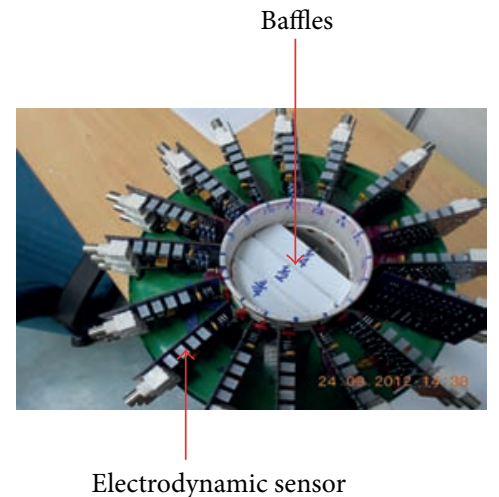

(b)

FIGURE 3: (a) Typical electrodynamic sensor on the printed circuit board and (b) sensors installed at the test point of pipeline showing baffling arrangement.

[17]. Figure 3(a) shows an electrodynamics sensor system with its conditioning circuit mounted on a printed circuit board. The electrode is a silver steel rod on the left of Figure 3(a), while Figure 3(b) shows the sensors installed at the test point of a pipeline showing the baffling arrangement used to simulate different flow profiles. When installed, the metal electrodes detect the charges of the moving particles. The detected charges are transduced and amplified by the amplifier, rectified by the precision rectifier, and filtered by the low pass filter.

2.2. The Sensor Configuration. In modeling of the system equation, a schematic diagram of the sensor configurations as shown in Figure 4 is made, in which the 16 electrodynamic sensors are equally spaced on the circumference of the pipeline around the sensing plane.

\subsection{Discretization of the Sensing Domain into Finite Elements.} The FEM involves subdivision of a problem domain into many subdivisions and each of the subdivisions is call the "finite-element" or "computational mesh" [18, 19]. In this paper, the mesh elements are the image pixels of the tomography system. Figure 5(a) shows a block diagram of pipe section with the cross-section meshed. The (b) part of the figure also shows a meshed domain with 1371 triangular elements and 16 sensors positioned equidistance from each other on the pipeline circumference. The (c) part of the figure is plot of central coordinates of the mesh elements.

2.4. The System Equation Modelling. The mathematical modelling involves the formulation of a relationship between the electrostatic fields due to the charges in each of the domain's mesh elements and the sensor electrodes as shown in the model in Figure 6. The model in Figure 6(a) shows a pipe section in $3 \mathrm{D}$, with sensor's cross-section and a sample of domain mesh element being exaggerated. The (b) part of Figure 6 shows an element of the domain showing the lines of electric flux due to the enclosed charges $\sigma A_{e}$ in Coulombs. According to Green et al. [20], the presence of charge/s at any location in a pipe cross-section affects the output of all sensor arrays installed around the sensing point of the pipeline. However, the equations are modelled to quantify the effects of the charges at any of the elements in the sensing zone. In deriving the equation, it is assumed that the particles move parallel to the pipe's $Z$-axis. Consider the model in Figure 6(a) of a pipeline with radius $r_{p}$ and a pin electrode sensor of radius $r_{s}$ installed around the pipeline circumference. The triangle adc is on the $Z$ plane while the triangle abc is on the $X-Y$ plane. The figure also shows a sample of a domain element exaggerated with central Cartesian coordinates $e_{i}\left(x_{i}, y_{i}\right)$ and surface area $A_{e}$ laying on 


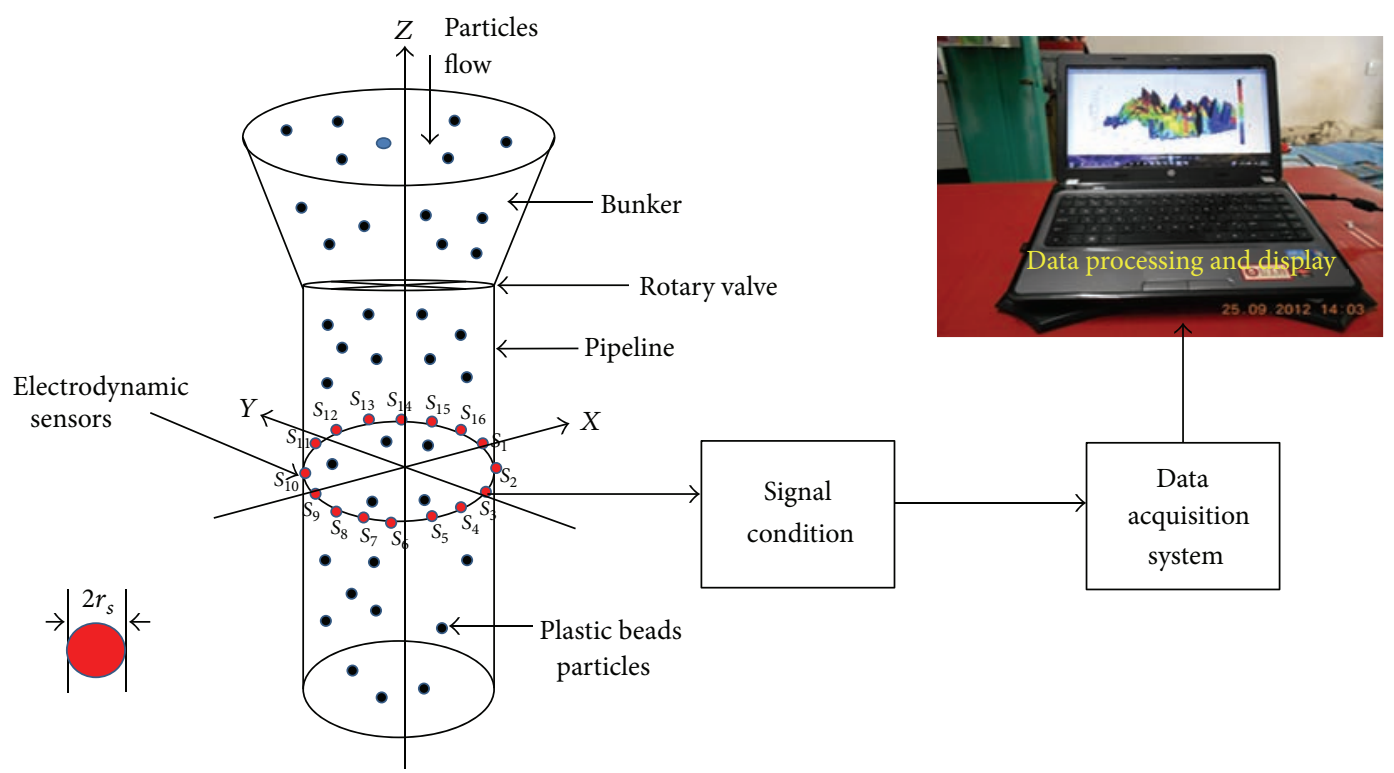

(a)

(b)

FIGURE 4: The block diagram of electric charge tomography system: (a) sensor cross-section dimension and (b) tomography system.

the same plane with the equally spaced sensors whose central Cartesian coordinates are also $S_{n}\left(x_{n}, y_{n}\right)$.

According to the Gauss law $[18,21]$, the electric charge produces an electric field, and the flux of the electric field passes through the surface that enclosed the charges and is mathematically stated as follows:

$$
\oint_{s} E \cdot \hat{n} d a=\frac{q^{\text {enclosed }}}{\varepsilon_{o}} .
$$

Implicitly, the electric field on the surface of each element on the sensing zone, due to the electric charges enclosed in the domain element, is given by

$$
E_{e i}=E=\frac{q^{\text {enclosed }}}{A_{e} \varepsilon_{o}},
$$

where $E$ is the electric field, $A_{e}$ is the surface area of the mesh element $\left(\mathrm{m}^{2}\right), q^{\text {enclosed }}$ is the total enclosed charges in the element in (Coulombs), $\varepsilon_{o}$ is the permittivity of the free space $\left(\mathrm{C}^{2} \mathrm{~N}^{-1} \mathrm{~m}^{-2}\right)$, and $E_{e i}$ is the electric field on the element surface $\left(\mathrm{NC}^{-1}\right)$. Assuming the electric field is isotropically radiated from the element, the radial component of the electric field that interrogates the sensors placed on the circumference of the pipeline from the element $e_{i}$ is given by

$$
E_{r}=E_{e i} \cos \theta=\frac{q^{\text {enclosed }}}{A_{e} \varepsilon_{o}} \cos \theta,
$$

where $\theta$ is the angle between the lines a $\bar{c}$ and a $\bar{d}$ in a triangle acd of Figure 6 for a given element $e_{i}$ and sensor $S_{n}$. According to Coulomb's law, the effect of the electric field at a point of distance $R$, due to electric field intensity at a reference point, is inversely proportional to the square of the distance between the points [22]. Therefore, (3) can be rewritten as follows:

$$
E_{r}=\frac{q^{\text {enclosed }}}{A_{e} \varepsilon_{o} R^{2}} \cos \theta \text {. }
$$

Consider Figure 6 again; the electric field source reference point is the centre of the exaggerated element, while the terminal reference point is the centre of the installed sensors, as can be seen in triangle abc of Figure 6(a). The distance $R$ is shown as "ac" in the figure. Therefore, replacing $R$ with "ac," (4) is now

$$
E_{r}=\frac{q^{\text {enclosed }}}{A_{e} \varepsilon_{o}(\mathrm{ac})^{2}} \cos \theta
$$

The quantity of the electric field interrogating the sensor is a fraction of the total electric field radiated from each element, which is called the sensor interrogation factor $K_{s}$ expressed as follows:

$$
K_{s}=\frac{A_{s}}{A_{p}}=\frac{r_{s}}{4 r_{p}},
$$

where $r_{s}$ is the sensor radius, $r_{p}$ is the pipeline cross-section radius, $A_{s}$ is $\pi r_{s}^{2}$ which is the sensor's cross-sectional area, and $A_{p}$ is $4 \pi r_{p} r_{s}$ the pipe strip surface area on which sensors are installed. Therefore, the actual electric field $E_{s}$ interrogating the sensor due to charges enclosed in each element on the sensing zone can be obtained by multiplying (5) and (6) to yield

$$
E_{s}=E_{r} K_{s}=\frac{r_{s} q^{\text {enclosed }}}{4 A_{e} r_{p}(\mathrm{ac})^{2} \varepsilon_{o}} \cos \theta .
$$

The charges induced by electrostatic sensor are the total electric flux captured by the sensor and it is the surface 


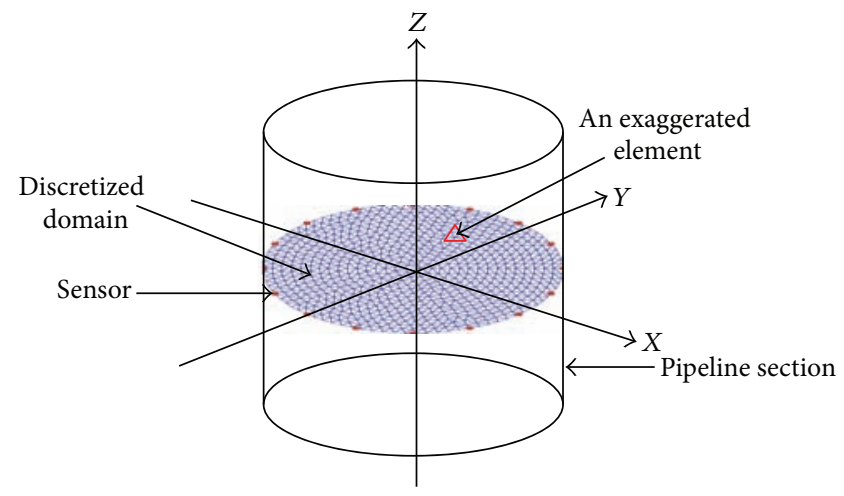

(a)

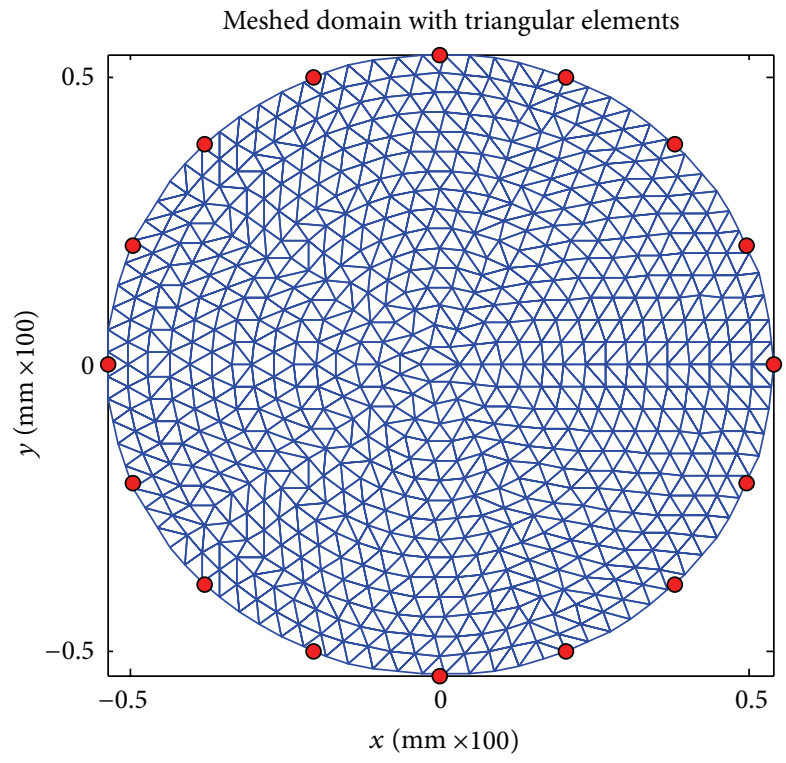

(b)

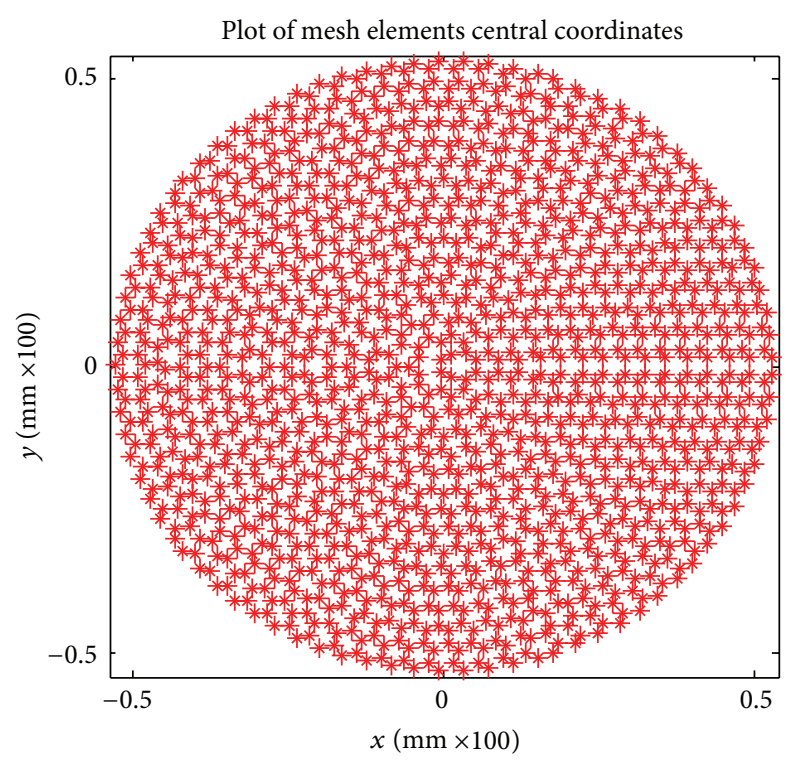

(c)

FIGURE 5: (a) Block diagram of the meshed pipeline cross-section with the 16 sensors installed, (b) programmatically meshed domain, and (c) domain elements' central coordinates plot.

integral of the electric flux that reaches the sensors [23]. Therefore, the electric field $E_{s}$ that reaches the sensor induces electric charges $Q_{s}$ on the sensor which can be expressed as follows:

$$
\begin{aligned}
-Q_{s} & =\varepsilon_{o} \oint_{s} E_{s} d A_{s}=\varepsilon_{0} \oint_{s} \frac{r_{s} q^{\text {enclosed }}}{4 A_{e}(\mathrm{ac})^{2} r_{p} \varepsilon_{o}} \cos \theta d A_{s} \\
& =\frac{r_{s} A_{s} q^{\text {enclosed }}}{4 A_{e}(\mathrm{ac})^{2} r_{p}} \cos \theta .
\end{aligned}
$$

The negative sign on the left-hand side of (8) is due to the movement of electrostatic charges from the element to the sensor. The value of $\cos \theta$ can be calculated trigonometrically from the triangles "adc" and "abc" of Figure 6 in terms of the element's and sensor's central coordinates as follows:

$$
\begin{gathered}
\cos \theta=\frac{\mathrm{ac}}{\mathrm{ad}}, \\
\mathrm{ac}=\left(\left(x_{n}-x_{i}\right)^{2}+\left(y_{n}-y_{i}\right)^{2}\right)^{1 / 2}, \\
\mathrm{ad}=\left((\mathrm{ac})^{2}+(\mathrm{cd})^{2}\right)^{1 / 2} .
\end{gathered}
$$

But

$$
(\mathrm{ac})^{2}=\left(x_{n}-x_{i}\right)^{2}+\left(y_{n}-y_{i}\right)^{2},
$$

where $c d$ is $r_{s}$ (the sensor electrode radius), ac is the distance from the centres of each reference element in the domain to the centre of each of the sensing pin electrodes, and $d A_{s}$ is the sensor cross-section deferential area. Other parameters of the equation are $x_{i}, y_{i}$ which are the $x$ and $y$ coordinates of the centre of the $i$ th element; $x_{n}$ and $y_{n}$ are also the $x$ 


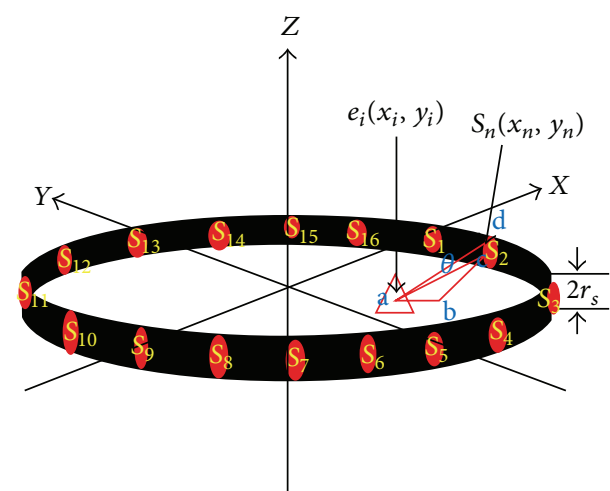

(a)

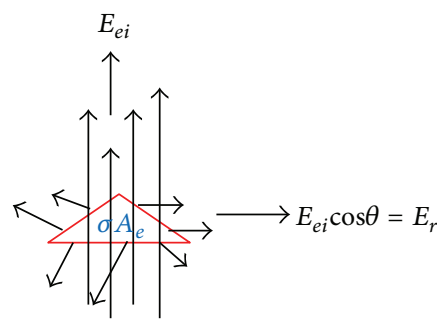

(b)

FIGURE 6: The model pipeline strip section: (a) pipe strip cross-section and (b) domain element [13].

and $y$ coordinates of the $n$th sensor, while $n$ and $i$ are the positive integers denoting sensors' and elements' numbers, respectively. Equations (9a)-(9d) were substituted into (8) and simplified to give (10). Equation (10) is the expression for electric charges induced in the sensor $n$ due to the enclosed electrostatic charges in element $i$ of the domain. Consider

$$
\begin{aligned}
Q_{S_{n}}=- & \left(\pi r_{s}^{3} q^{\text {enclosed }}\right) \\
\times & \left(4 A_{e} r_{p} \sqrt{\left(x_{i}-x_{n}\right)^{2}+\left(y_{i}-y_{n}\right)^{2}}\right. \\
& \left.\times \sqrt{\left(x_{i}-x_{n}\right)^{2}+\left(y_{i}-y_{n}\right)^{2}+r_{s}^{2}}\right)^{-1} .
\end{aligned}
$$

Assume spatially distributed charged particles flowing through a pipeline cross-section and the flowing particles carry charges whose area-charge distribution density around the pipe cross-section is $\sigma \mathrm{Cm}^{-2}$. The particles' charge, enclosed in each of the domain mesh elements is given by (11)

$$
q^{\text {enclosed }}=A_{e} \sigma \mathrm{C}
$$

$A_{e}$ is the surface area of each of the mesh elements in $\mathrm{m}^{2}$ and $\mathrm{C}$ is the unit of electric charge called "Coulombs"

$$
\begin{aligned}
Q_{s_{n}}= & -\left(\pi r_{s}^{3} \sigma\right) \\
& \times\left(4 r_{p} \sqrt{\left(x_{i}-x_{n}\right)^{2}+\left(y_{i}-y_{n}\right)^{2}}\right. \\
& \left.\times \sqrt{\left(x_{i}-x_{n}\right)^{2}+\left(y_{i}-y_{n}\right)^{2}+r_{s}^{2}}\right)^{-1},
\end{aligned}
$$

where $Q_{s}$ is the sensor induced charges in Coulombs. Due to the influence of electric field resulting from the charge carried by the flowing particles, there exists a current $I_{s}$ flowing through the sensor expressed as follows:

$$
I_{s}=-\frac{d Q_{s}}{d t}
$$

Suppose the particles move at a group velocity of $\mathrm{Vms}^{-1}$; then the time taken to travel through the diametric distance of the sensor of radius $r_{s}$ is

$$
t=\frac{2 r_{s}}{V}(s) \Longrightarrow d t=\frac{2}{V} d r_{s} .
$$

Substitution of $Q_{s}$ and $d t$ is made into (13) and simplified using the MUPAD package of MATLAB to obtain (15). Therefore, the induced current $\left(I_{s}\right)$ in sensor $n$ due to the electric field from the charges on element $i$ of the domain was obtained as follows:

$$
I_{s}=-\frac{V\left(\left(3 \pi \sigma r_{s}^{2} / 4 r_{p} \sigma_{2} \sqrt{\sigma_{1}}\right)-\left(\pi \sigma r_{s}^{4} / 4 r_{p} \sigma_{2} \sigma_{1}^{3 / 2}\right)\right)}{2},
$$

where $\sigma_{1}=\left(x_{i}-x_{n}\right)^{2}+\left(y_{i}-y_{n}\right)^{2}+r_{s}^{2}, \sigma_{2}=$ $\sqrt{\left(x_{i}-x_{n}\right)^{2}+\left(y_{i}-y_{n}\right)^{2}}$.

With the installed sensors, the electrostatic charges carried by the moving particles can be captured as data. By an appropriate algorithm, the parameters of interest of the moving particles (such as velocity, mass flow rate, particle sizes, and concentration profile) can be determined. MAT$\mathrm{LAB}$ codes were designed to compute the coordinates of the element nodes from which elements' central coordinates $x_{i}$ and $y_{i}$ were obtained, whereby space coordinates interpolation techniques of FEM were applied [24], using the Langrage shape function of (16). Consider

$$
\begin{array}{r}
x_{i}=x_{1}^{e i} N_{1}+x_{2}^{e i} N_{2}+x_{3}^{e i} N_{3}=\sum_{j=1}^{3} x_{j}^{e i} N_{j}, \\
\left\{\begin{array}{c}
j=\text { nodes of element } e_{i} \\
N=\text { shape function of } e_{i}
\end{array}\right\}, \\
y_{i}=y_{1}^{e i} N_{1}+y_{2}^{e i} N_{2}+y_{3}^{e i} N_{3}=\sum_{j=1}^{3} y_{j}^{e i} N_{j} .
\end{array}
$$

In meshing of the domain, the main objective is to have a good mesh element where shapes of each element should be square (quadrilateral shape) or equilateral-triangle (triangular shape) [24]. The obtained central coordinates of elements 


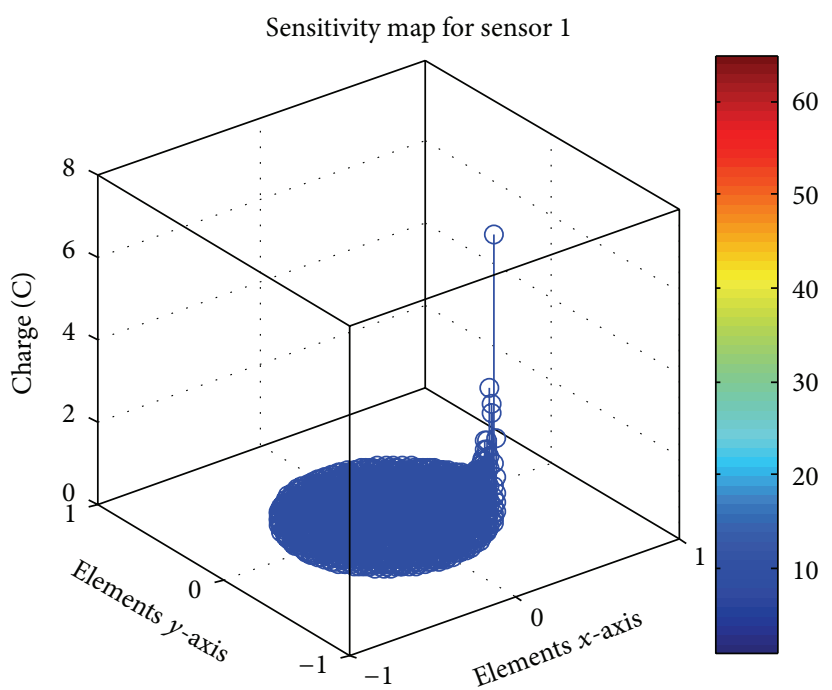

(a)

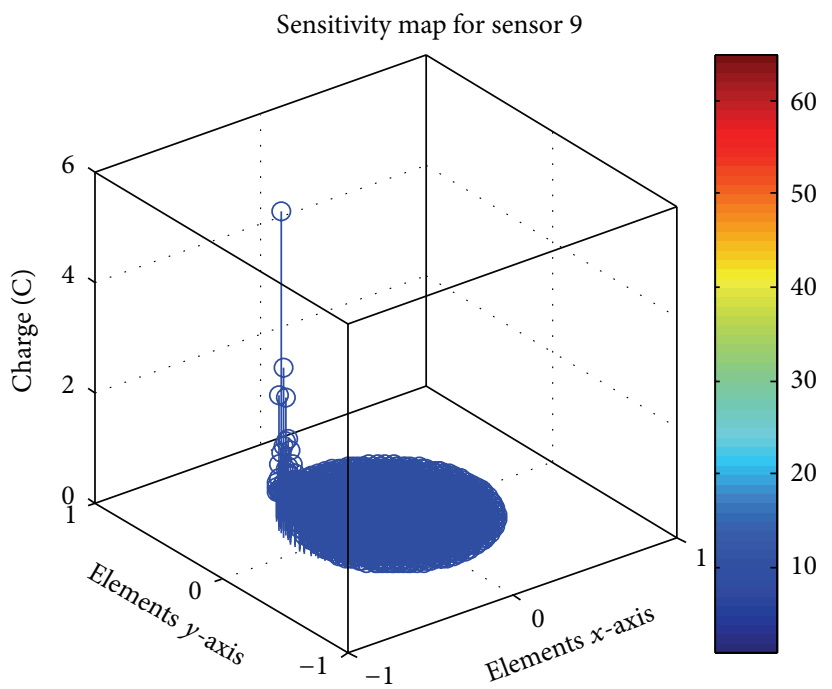

(c)

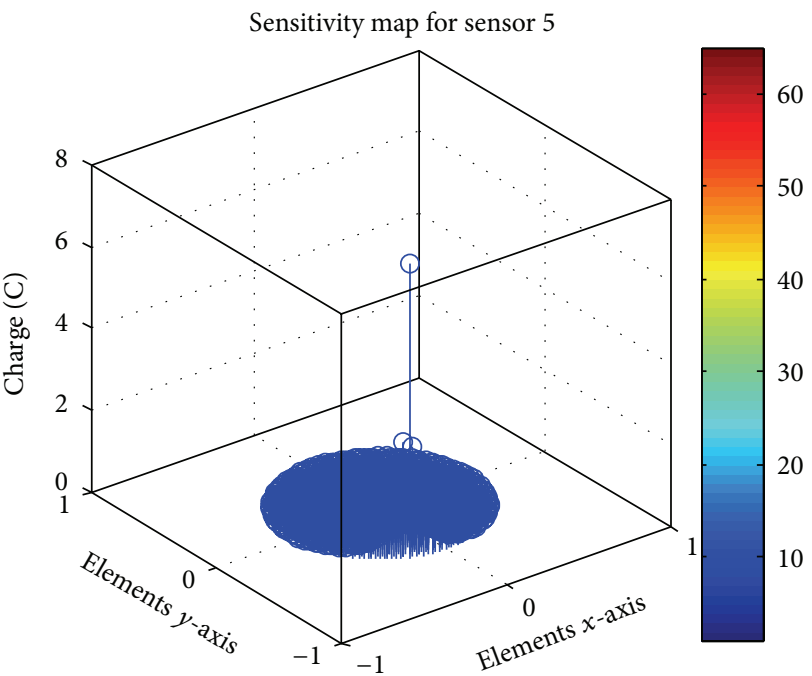

(b)

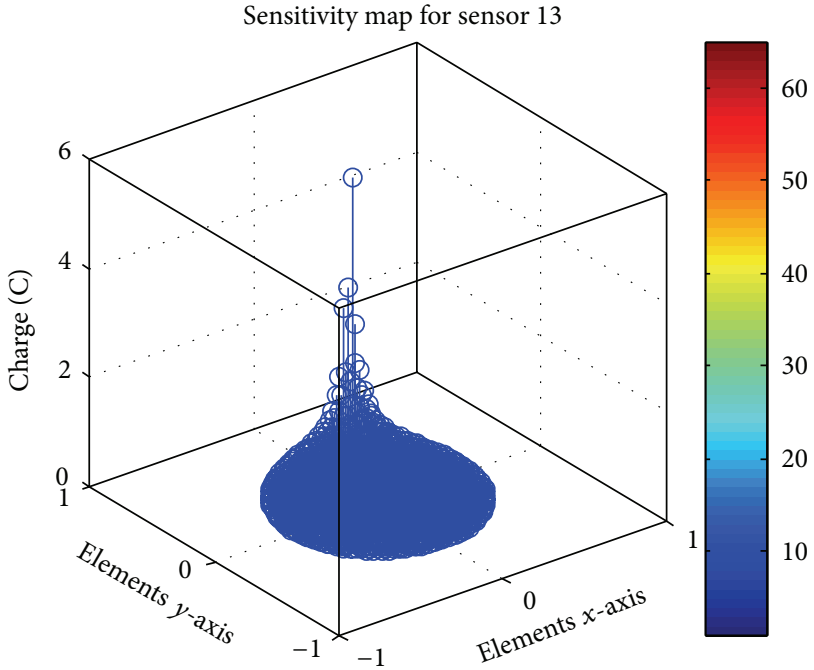

(d)

FIGURE 7: Sensitivity maps for selected sensors 1, 5, 9, and 13.

and sensors were used to derive the system equation (12). However triangular mesh elements were structurally generated in the pipe cross-sectional area as shown in Figure 5 by the use of the designed MATLAB codes. The generated elements have known nodes coordinates, from which their central coordinates are computed using (16) as shown in Table 1. The sensors central coordinates are also shown in Table 2.

\section{The Electric Charge Tomographic Image Reconstruction}

As mentioned earlier, one of the most important procedures in the concentration profiling or tomography image reconstruction is the generation of sensitivity matrix $S$ of the system. Using the system equation (12), the sensitivity matrix $S$ of $[M \times N]$ as shown in Table 3 , of size $[1371 \times 16]$ for the 16-sensor system, was generated. In the generation of the sensitivity matrix, parameters of the experimental equipments were used, which are the conveying pipeline radius $\left(r_{p}\right)$ of $54 \mathrm{~mm}$, the sensor electrode radius $\left(r_{s}\right)$ of $2.4 \mathrm{~mm}$, and an assumed equal charge density distribution $(\sigma)$ of $1 \mathrm{Cm}^{-2}$ across the pipe cross-section. The sensitivity maps of the system are presented in Figure 7 for some selected sensors as an example, that is, Figure 7(a) sensor 1, Figure 7(b) sensor 5, Figure 7(c) sensor 9, and Figure 7(d) sensor 13. It can be seen from the sensitivity maps that the sensors have the ability to correctly sense the distribution of charges in the pipeline cross-section, based on the map pattern where the charge sensitivity magnitude peaks near the sensor and diminishes along the horizontal plane. For the concentration profiling, all the data captured by the 16 sensors are usually used.

One of the problems of electrodynamic tomography imaging of particles in a pipeline is the weak signal response 
TABLE 1: Extract from elements central Cartesian coordinates.

\begin{tabular}{lcccccccc}
\hline \multirow{2}{*}{ Coordinates } & \multicolumn{9}{c}{ Element $e_{i}$ and its central Cartesian coordinate } \\
& $e_{1}$ & $e_{2}$ & $e_{3}$ & $e_{4}$ & $e_{5}$ & $e_{6}$ & - & $e_{1371}$ \\
\hline$x_{i}$ & -0.21 & -0.30 & -0.43 & -0.28 & -0.13 & 0.15 & - \\
$y_{i}$ & -0.13 & -0.17 & -0.25 & $-5.20 E-18$ & -0.06 & 0.29 & - \\
\hline
\end{tabular}

TABLE 2: Sensors central Cartesian coordinates.

\begin{tabular}{cccccccccccccccccccccc}
\hline \multirow{2}{*}{ Coordinates } & \multicolumn{11}{c}{ Sensor $S_{n}$ and its central Cartesian coordinate } \\
& $S_{1}$ & $S_{2}$ & $S_{3}$ & $S_{4}$ & $S_{5}$ & $S_{6}$ & $S_{7}$ & $S_{8}$ & $S_{9}$ & $S_{10}$ & $S_{11}$ & $S_{12}$ & $S_{13}$ & $S_{14}$ & $S_{15}$ & $S_{16}$ \\
\hline$x_{n}$ & 0.50 & 0.38 & 0.21 & $-9.92 E-17$ & -0.21 & -0.38 & -0.50 & -0.54 & -0.50 & -0.38 & -0.21 & $3.31 E-17$ & 0.21 & 0.38 & 0.50 & 0.54 \\
$y_{n}$ & -0.21 & -0.38 & -0.50 & -0.54 & -0.50 & -0.31 & -0.21 & $6.61 E-17$ & 0.21 & 0.38 & 0.50 & 0.54 & 0.50 & 0.38 & 0.21 & 0 \\
\hline
\end{tabular}

TABLE 3: Extract from the sensitivity matrix.

\begin{tabular}{lccccc}
\hline \multirow{2}{*}{ Elements $\left(e_{i}\right)$} & $S_{1}$ & $S_{2}$ & $S_{3}$ & - & $S_{16}$ \\
\hline$e_{1}$ & 0.098 & 0.109 & 0.127 & - & 0.092 \\
$e_{2}$ & 0.098 & 0.110 & 0.131 & - & 0.091 \\
$e_{3}$ & 0.084 & 0.095 & 0.116 & - & 0.077 \\
$e_{4}$ & 0.097 & 0.103 & 0.113 & - & 0.096 \\
- & - & - & - & - & - \\
$e_{1371}$ & 0.657 & 0.983 & 0.282 & & 0.247 \\
\hline
\end{tabular}

TABLE 4: Extract from the filtered normalized sensitivity matrix.

\begin{tabular}{lccccc}
\hline \multirow{2}{*}{ Elements $\left(e_{i}\right)$} & \multicolumn{5}{c}{ Sensors $\left(S_{n}\right)$} \\
& $S_{1}$ & $S_{2}$ & $S_{3}$ & - & $S_{16}$ \\
\hline$e_{1}$ & 0.72 & 0.66 & 0.52 & - & 0.74 \\
$e_{2}$ & 0.72 & 0.65 & 0.50 & - & 0.75 \\
$e_{3}$ & 0.84 & 0.75 & 0.57 & - & 0.88 \\
$e_{4}$ & 0.72 & 0.69 & 0.58 & - & 0.71 \\
- & - & - & - & - & - \\
$e_{1371}$ & 0.11 & 0.07 & 0.23 & - & 0.28 \\
\hline
\end{tabular}

around the centre of the pipe cross-section. To address the problem, Rahmat et al. [25] proposed matrix filtering technique represented by

$$
F=\left\{\frac{S_{\max }}{S_{i}}\right\}
$$

where $F$ is the filtered matrix, $S_{\max }$ is the maximum value of the pixel of the sensitivity matrix $S$, and $S_{i}$ is the $i$ th pixel value of $S$. Using (17), the sensitivity matrix of Table 3 was filtered and the extract from the filtered matrix is shown in Table 4.

The well-known method called linear back projection (LBP) normally employed in the electric charge tomography imaging is based on the fact that the image is reconstructed by redistributing electric charges carried by the particles measured as voltage through the electrodynamics sensors along each projection [25] through matrix inverse operation. But in case of the PRD method, the captured voltage from each sensor is distributed proportionately to the value of the electric charge of each element of the domain as sensed by the sensor as per the system's sensitivity matrix. The advantages of the PRD over the LBP are that PRD does not require the system matrix to be symmetric and can be used for any matrix shape while LBP requires only square matrix. Another advantage is that PRD does not require inversion of matrix as being the requirement in other existing methods of the electric charge tomography imaging such as the singular value decomposition (SVD) reported in [26], LBP, and FBP. To use the data for the four flow regimes in the reconstruction of the concentration profiles using the traditional LBP method, the generated sensitivity matrix is made into a square and symmetric matrix through transpose operation. The superiority of the PRD over the other methods is that it can be used for any shaped matrix, not necessarily the square matrix. This property of the PRD has been demonstrated in the paper. This is because the resulting data matrix from the finite element computation is rectangular and can be made symmetric and square [27] through simple transposition as per (18) which is called the matrix compression. In (18), Mc is the compressed [16 $\times 16]$ matrix, $F$ is main sensitivity matrix of size $[1371 \times 16]$ whose elements are the charge magnitude on each element as sensed by each of the 16 sensors, and $F^{\prime}$ is the transpose of $F$. The compressed result was also used for the concentration profile of the moving particles:

$$
M_{c}=F^{\prime} F .
$$

\section{Experimentation}

4.1. Experimental Conditions. According to Yan [6], the electrodynamic sensing technique offers the cheapest and simplest means of measuring solids flows in pipes. Because the sensors respond only to moving solids in the pipe and that the concentration data is highly immune from solid accretions that affect other technologies such as electrical capacitance tomography (ECT), which uses permittivity distribution within the sensing zone, the major problem in applying this sensing technique lies in relating the measured solids concentration to the magnitude of the charge signal in both AC and DC methods. The magnitude largely depends on physical properties of the particles which are size, shape, distribution, conductivity, permittivity, chemical-composition, moisture content, and so on. Other properties of interest are the conditions of the conveying medium which include pipe size, pipe wall roughness, and line temperature. Other factors 


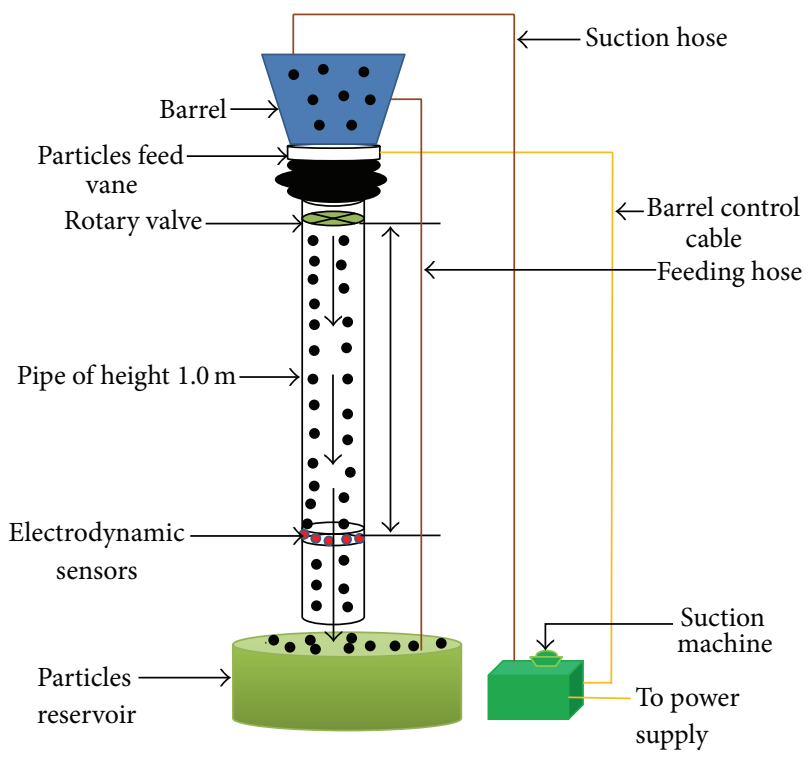

(a)

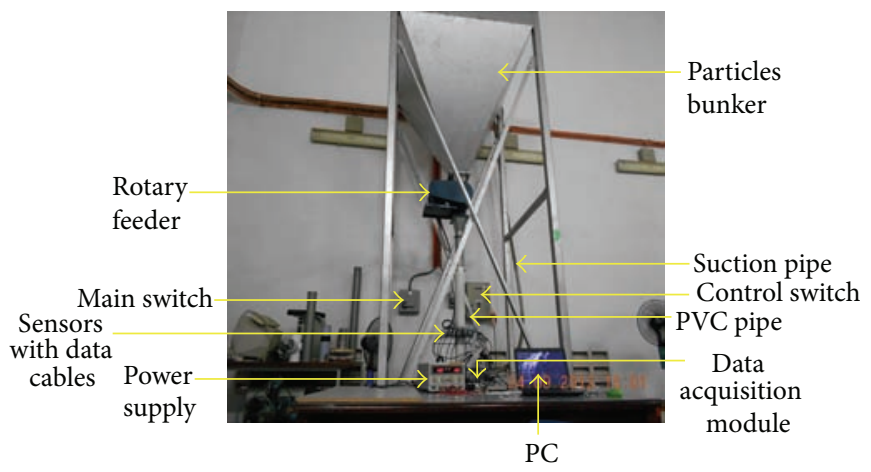

(b)

FIGURE 8: The experimental set-up: (a) block diagram and (b) real photograph during experimentation.

contributing to the magnitude of the charge signal are the concentration and velocity of the solid particles. In view of the above considerations, the experimental conditions were set and noted, which are plastic beads of the same material and uniform sizes used as the test particles at an average room temperature of $30^{\circ} \mathrm{C}$ and prevailing atmospheric condition. The gravity drop test rig was used to maintain constant velocity. A nonconducting Ply-Vinyl-Chloride (PVC) pipe was used as the test pipeline, to avoid discharge of charges on the moving particles through conducting pipeline. The particle discharge was maintained at a mass flow rate of $300 \mathrm{~g} / \mathrm{s}$. Figure 8 shows the experimental setup.

In order to evaluate the concentration profiling method described, several experiments were carried out using the sixteen electrodynamics sensors installed around the gravity dropped conveying pipeline as shown in Figure 8(b). The purpose of the experiment was to obtain data from each of the 16 sensors. The obtained data were used for the concentration profile of particles moving through the pipeline. The length between the rotary valve and the array of sensors was $1000 \mathrm{~mm}$ and the pipe internal diameter was $108 \mathrm{~mm}$. In carrying out the experiment, the plastic bead particles are transported to the bunker by a suction machine through suction hose. The mechanical rotary valve of the bunker releases the particles which move under gravity and the electrostatic charges carried by the moving particles are sensed by the 16 installed electrodynamic sensors. The particles are accelerated under gravity with a uniform acceleration due to gravity of $9.81 \mathrm{~ms}^{-2}$ along the pipe vertical axis. Electrification occurs due to rotary valve effect on the particles, frictional interaction among the particles, transporting gas, and the pipe wall during the conveying process. The process generates charges of the moving particles which in turn are captured by the electrodynamic sensory systems through induction. The induced charge is transduced into a voltage signal through conditioning circuit attached to the sensor electrode. The data in the form of voltage were used in the developed algorithm to reconstruct the particle concentration profile referred to as the tomographic image of the flowing particles. The computer system was used to capture the data via Keithley KUSB-3116 data acquisition module.

4.2. Algorithm of the Image Reconstruction. In the image reconstruction, PRD approach was employed in which the captured voltage from each sensor is distributed proportionately to each element's charge as sensed by each sensor calculated as per the generated system's sensitivity matrix.

In pro rata distribution, if $n$ people contributed $m=$ $k_{1}+k_{2}+\cdots+k_{n}$, amount of money to invest in a venture, from which the investment yielded a profit of $P$ amount, the profit sharing among the investors in pro rata [15] is as per (19). Consider

$$
P_{i}(n)=\frac{k_{i}}{\sum_{i=1}^{n} k_{i}} P,
$$

where $P_{i}(n)$ is the $i$ th person's share of the profit, $P$ is the total profit, and $k_{i}$ is the contribution of each investor. A similar approach was presented in Landweber's iteration method quoted in [26] where a simple linear approximation for forward problem is used in matrix form as in (20). Consider

$$
A \mathbf{x}=\mathbf{b},
$$

where $A$ is $[M \times N]$ normalized sensitivity matrix, in which $M$ is the image pixels (the total elements of the domain), $N$ is the number of electrodynamic sensors installed around the pipeline, $\mathbf{x}$ are the vectors of the image of the charge distribution of the domain, and $\mathbf{b}$ are the vectors which are the charges measured in the form of voltages from each of 


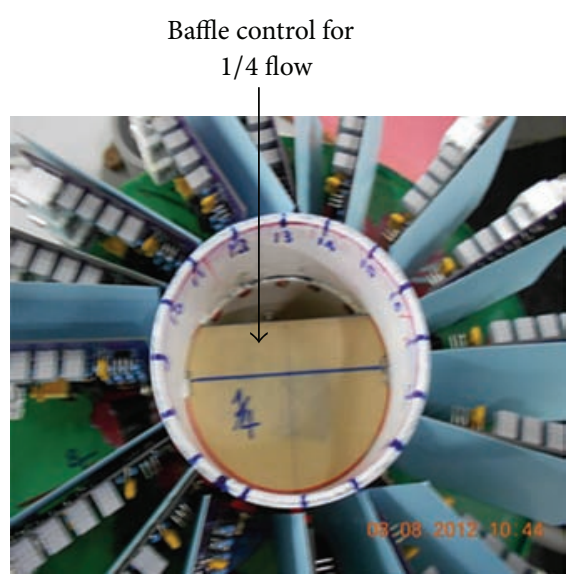

(a)

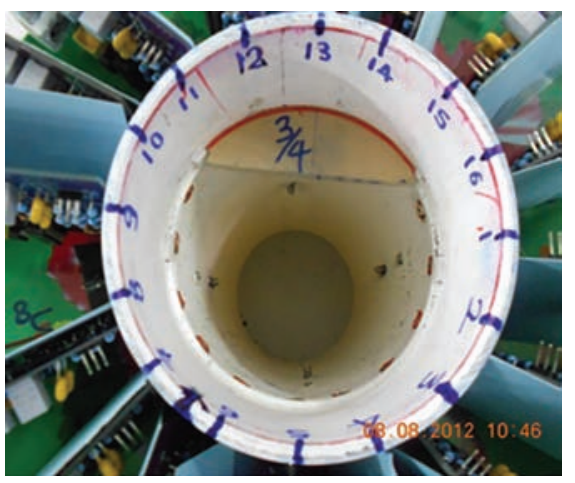

(c)

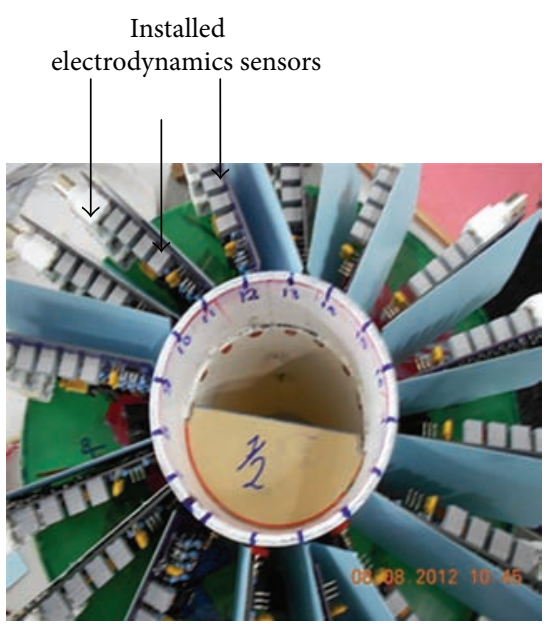

(b)

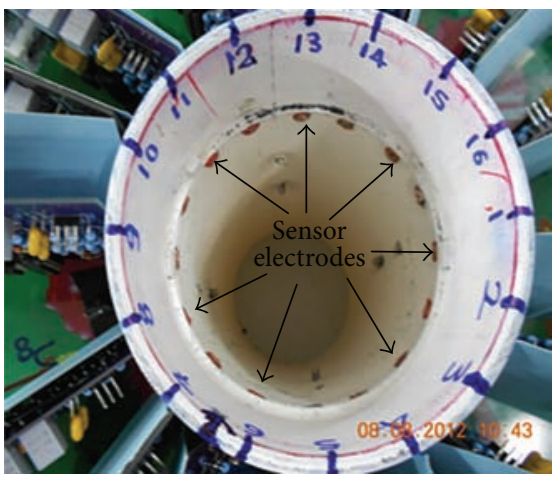

(d)

FIgURE 9: Particle flow control arrangement: (a) 1/4-flow regime, (b) 1/2-flow regime, (c) 3/4-flow regime, and (d) full-flow regime.

the 16 installed sensors via the data acquisition module. The sensitivity matrix is calculated using the system equation (12). The calculated sensitivity values are for $M$ elements and $N$ sensors arranged in a sensitivity matrix of $[M \times$ $N$ ], used to represent $M$ rows of 1371 elements and $N$ columns of 16 sensors. In the reported Landweber's method, a new normalized estimator matrix was obtained using (21), which is similar to the multiplication factor of the pro rata distribution of (19), which was obtained as

$$
S_{i, j}^{m}=\frac{S_{i, j}}{\sum_{i=1}^{m} S_{i, j}}
$$

Applying the PRD concept, each element contributed according to the value of the sensitivity matrix obtained from the system equation under the constant charge distribution across the pipe cross-section. The measured voltage obtained during different flow regimes was divided proportionately to the value of the sensitivity matrix on each element using (22). The main difference between Landweber's method and $\mathrm{PRD}$ is that Landweber's method requires invertible square matrix while the PRD does not. The Landweber iteration was designed to solve ill-posed problem in which invertible matrix is calculated from the iteration of the assumed invertible matrix by the use of the identity matrix $(I)$ which is square [28]. The extract from the pro rata matrix for the full-flow regime as an example is shown in Table 6. The data in Table 6 are based on the normalized values of the filtered sensitivity matrix. Consider

$$
A_{i, j}=\frac{S_{i, j}^{m}}{\sum_{i=1}^{m} S_{i, j}} V_{j}^{\prime} \quad\left\{\begin{array}{l}
i=1,2, \ldots m=1371, \\
j=1,2, \ldots n=16, \\
V_{j}=\text { voltage obtained from sensor } j .
\end{array}\right.
$$

\section{Results}

5.1. Data Captured. Experiments were carried out with plastic beads using the standard test rig shown in Figure 8(b). During the experiment, baffles of the flowing particles were made through artificially constricting the pipe flowing channel into four. The constrictions are $1 / 4$ (quarter)-flow, $1 / 2$ (half)-flow, 3/4 (three-quarter)-flow, and full-flow as shown in Figure 9. Each of the flow situations is called a flow regime. The 4 flow regimes arose from the fact that, four flow arrangements were made for the experimentation purpose.

Table 7 shows voltage magnitudes from the 16 sensors captured during experimentation on the artificially created four flow regimes and the bar chart of the voltages is presented in Figure 10, in which Figure 10(a) is voltage captured 


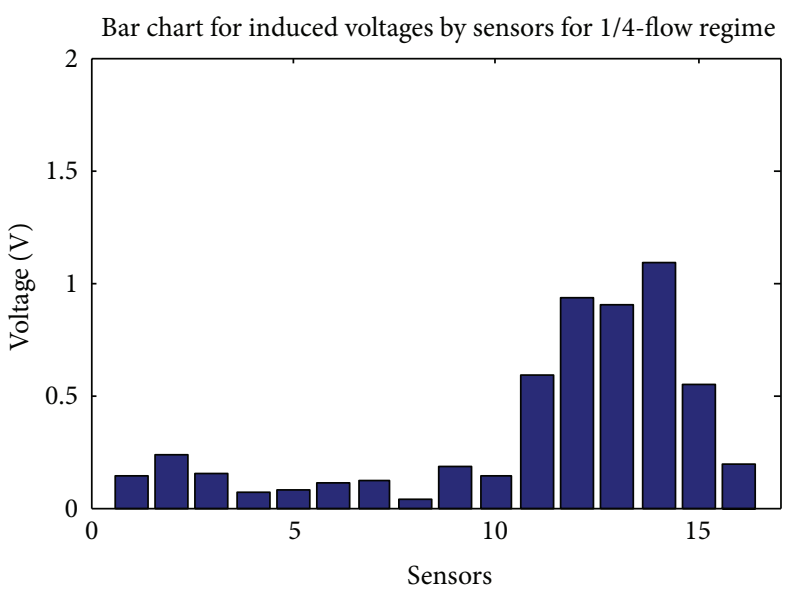

(a)

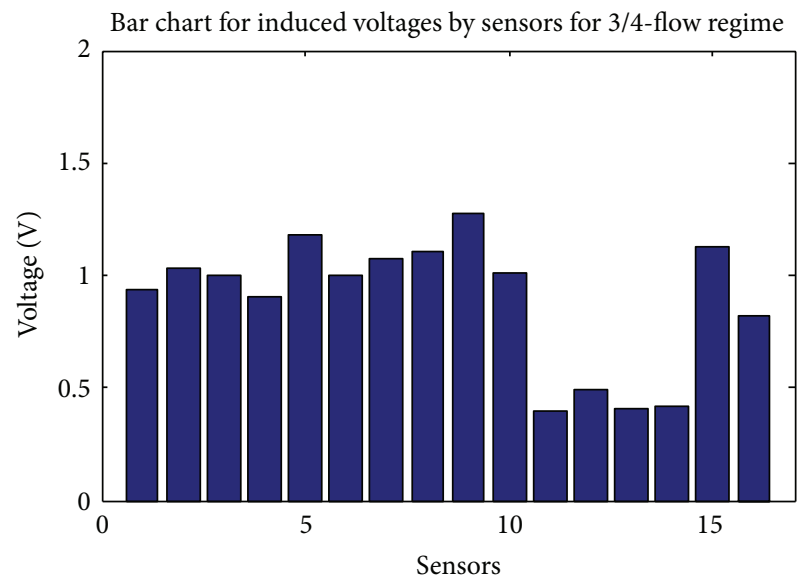

(c)

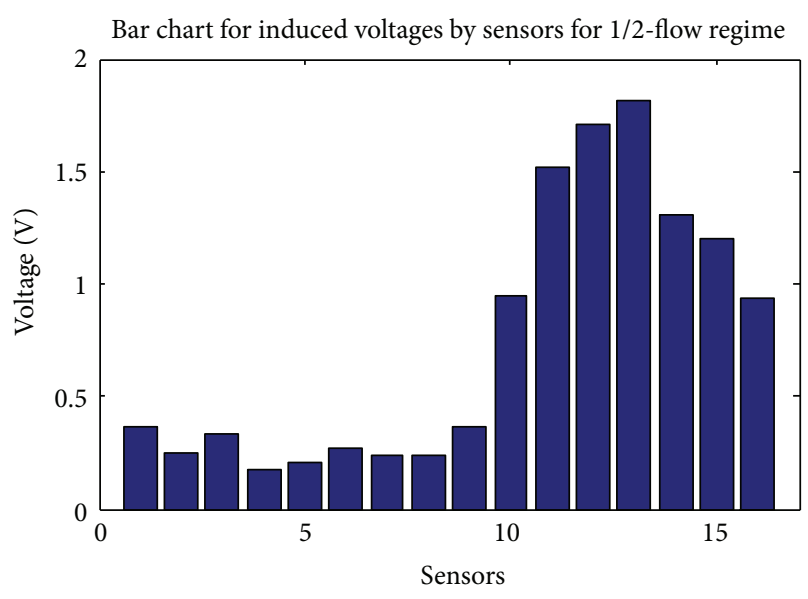

(b)

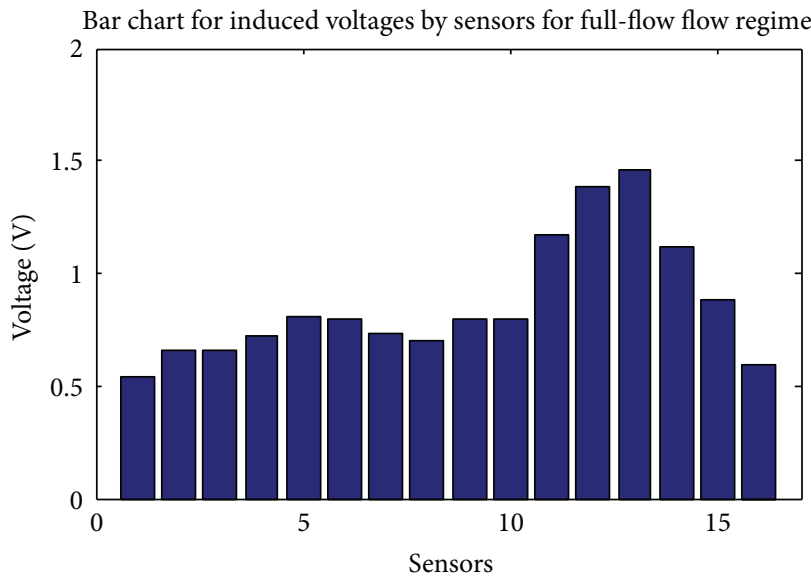

(d)

FIGURE 10: Bar charts of the voltages captured by each sensor at the four different flow regimes.

by each sensor during quarter-flow regime, Figure 10(b) voltage captured by each sensor during half-flow regime, Figure 10(c) voltage captured by each sensor during threequarter-flow regime, and Figure 10 (d) voltage captured by each sensor during full-flow regime experiment, respectively.

The captured voltages were used to generate the concentration profile data using the PRD techniques as per (22). To demonstrate the reconstruction of the concentration profile using the PRD in any shaped matrix, two image data are to be used for the reconstruction of the concentration profile. The first, is the triangular image matrix for the triangular elements of size $[1371 \times 16]$ obtained by the use of the sensitivity matrix of Table 4 . The second is the square image data obtained by compression of the sensitivity matrix to $[16 \times 16]$ through transposition as per (18) and then filtered as in Table 5. Therefore, the concentration profiles for the rectangular and square matrices using the PRD concept are presented in Figures 11 and 12 for the four different flow regimes. The data in Table 4 were also used to reconstruct the concentration profile in terms of the mesh elements and are presented in Figure 13.
TABLE 5: Extract from the compressed to $[16 \times 16]$ filtered sensitivity matrix.

\begin{tabular}{lccccc}
\hline Pixels & \multicolumn{5}{c}{ Sensors } \\
& 1 & 2 & 3 & - & 16 \\
\hline 1 & 1.057 & 2.041 & 2.705 & - & 2.048 \\
2 & 2.041 & 1.027 & 2.054 & - & 2.694 \\
3 & 2.705 & 2.054 & 1.189 & - & 3.233 \\
4 & 3.224 & 2.696 & 2.062 & - & 3.657 \\
- & - & - & - & - & - \\
16 & 2.048 & 2.694 & 3.233 & - & 1.124 \\
\hline
\end{tabular}

5.2. Photographs Captured during the Experimentation. The data captured in the form of voltages presented in Figure 10 shows that the concentration profile of the moving plastic beads shown in Figures 11 and 12 clearly represented the captured data for different flow regimes. There is good agreement between Figures 10 and 12 ((a1), (b1), (c1), and (d1)). It is worth noting that the reconstructed image or concentration profile is not smooth and is probably due to the nature of the feeder, which does not give a homogeneous particle distribution 


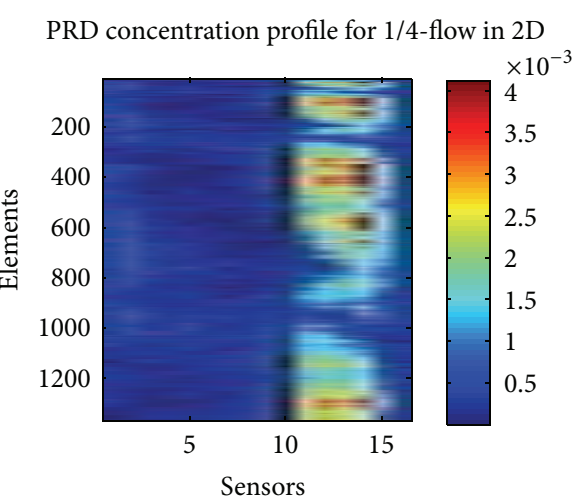

(a1)

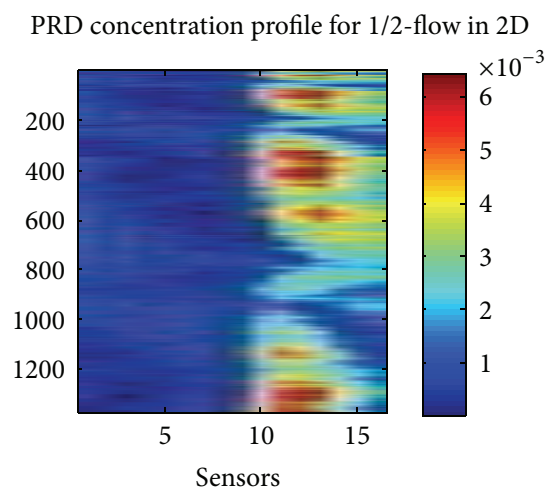

(b1)

PRD concentration profile for $3 / 4$-flow in $2 \mathrm{D}$

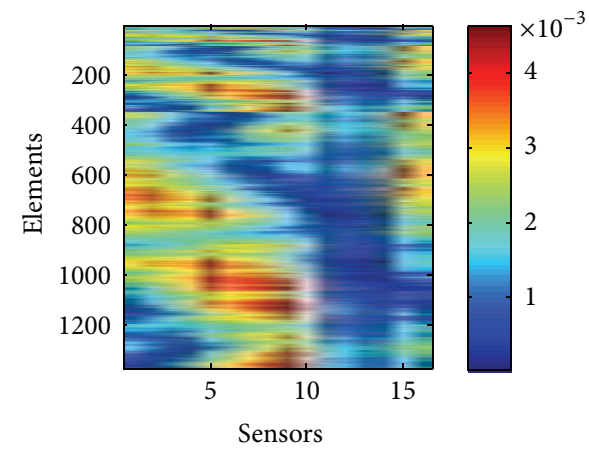

(c1)

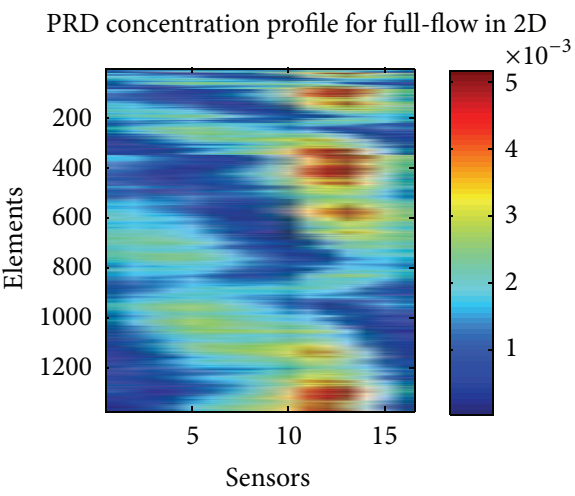

(d1)

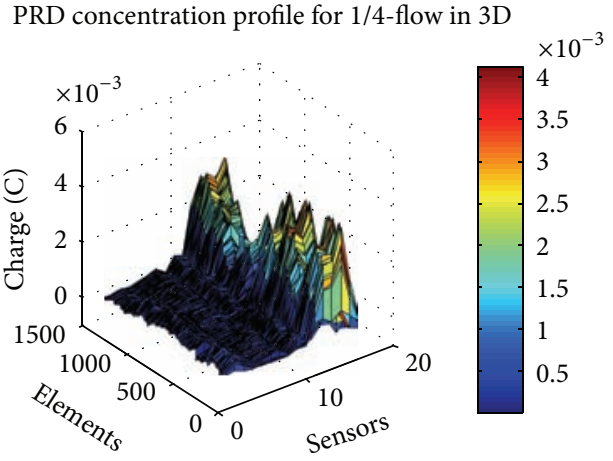

(a2)

PRD concentration profile for $1 / 2$-flow in 3D

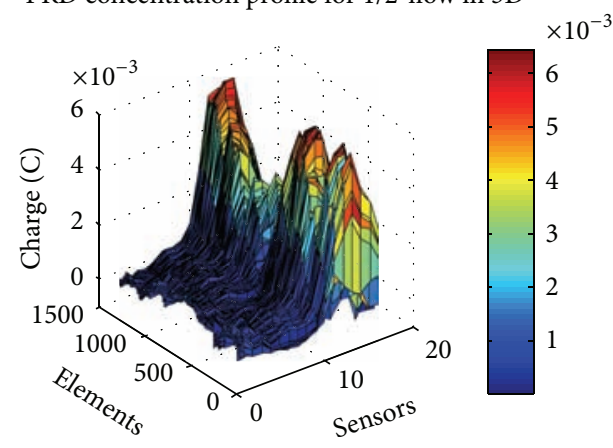

(b2)

PRD concentration profile for $3 / 4$-flow in 3D

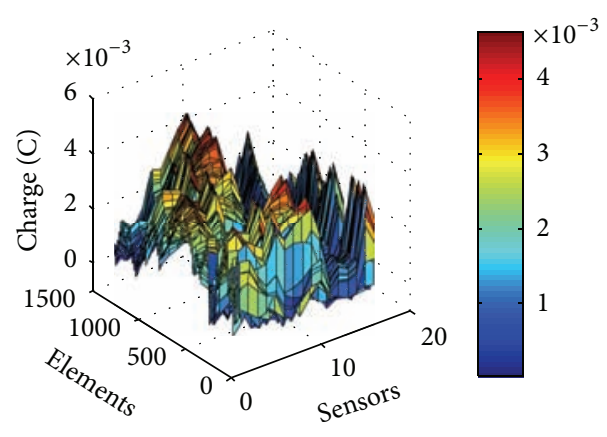

(c2)

PRD concentration profile for full-flow in 3D

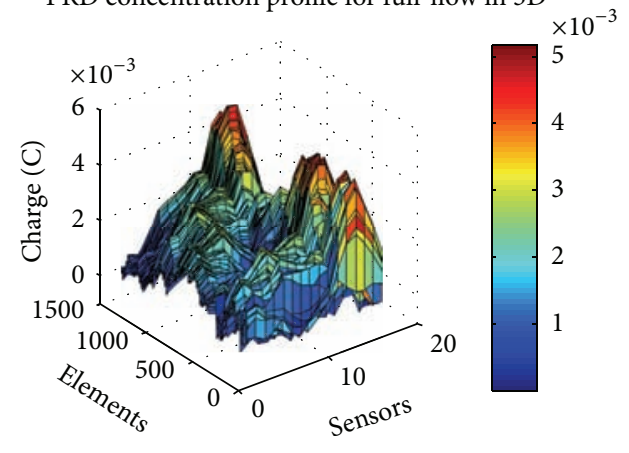

(d2)

FIGURE 11: Concentration profiles using the triangular matrix in 2D and 3D for the four flow regimes using PRDM. 


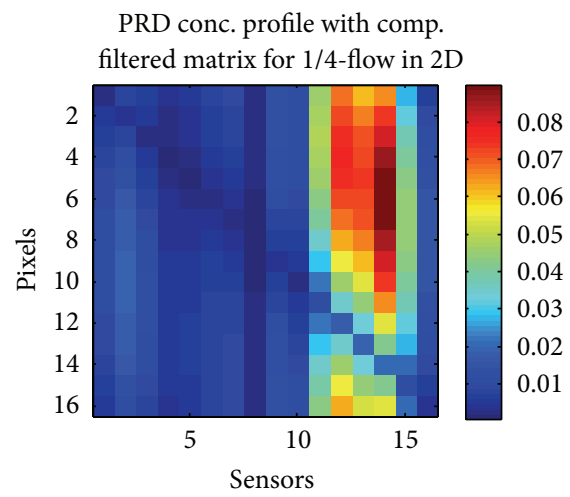

(a1)

PRD conc. profile with comp. filtered matrix for $1 / 2$-flow in $2 \mathrm{D}$

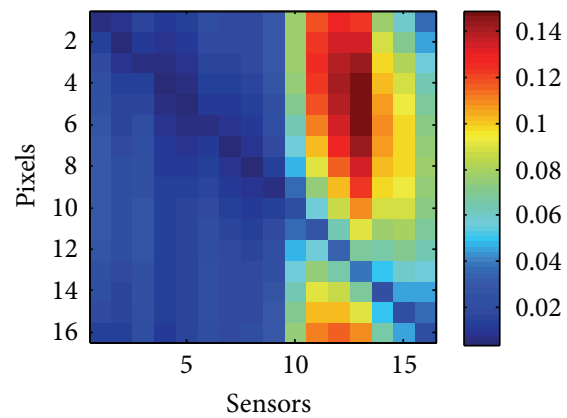

(b1)

PRD conc. profile with comp. filtered matrix for $3 / 4$-flow in $2 \mathrm{D}$

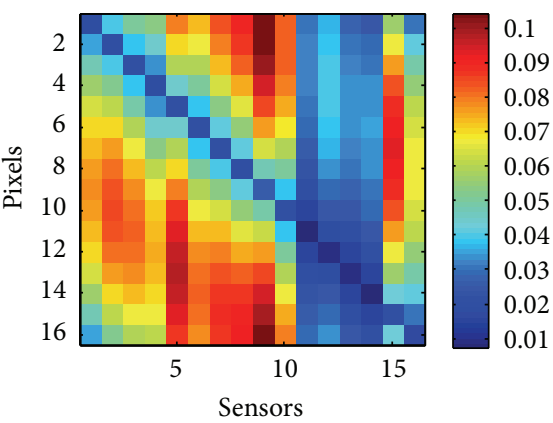

(c1)

PRD conc. profile with comp.

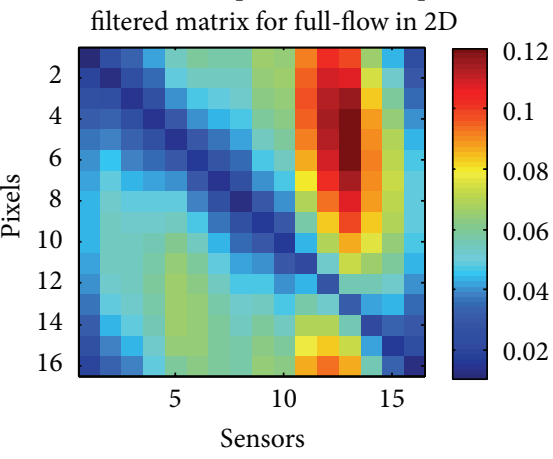

(d1)
PRD conc. profile with comp.

filtered matrix for $1 / 4$-flow in $3 \mathrm{D}$

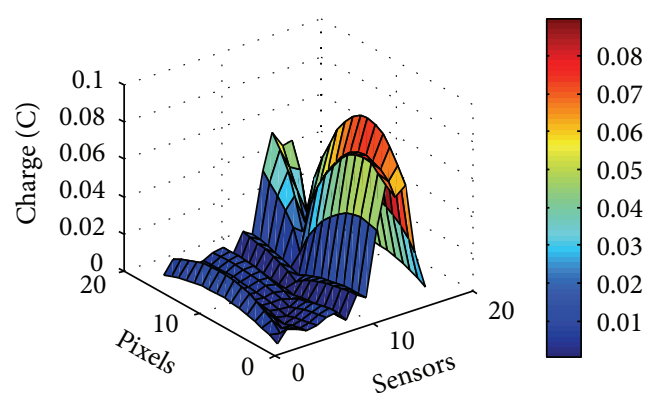

(a2)

PRD conc. profile with comp.

filtered matrix for $1 / 2$-flow in $3 \mathrm{D}$

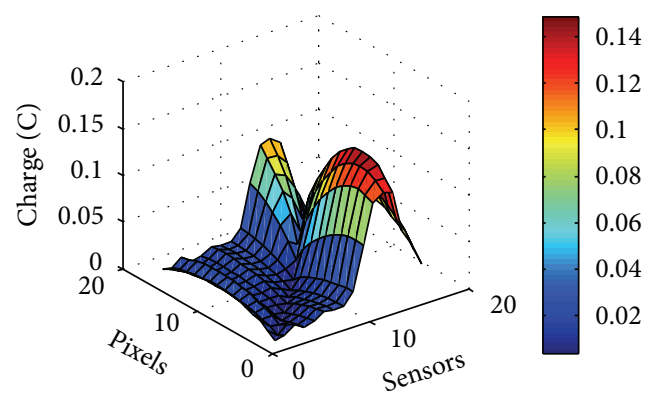

(b2)

PRD conc. profile with comp. filtered matrix for $3 / 4$-flow in $3 \mathrm{D}$

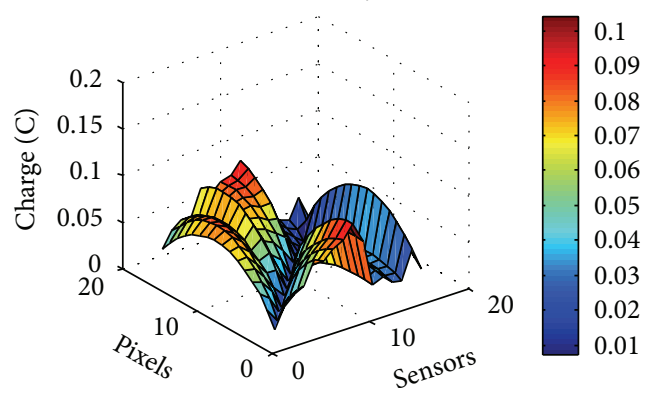

(c2)

PRD conc. profile with comp. filtered matrix for full-flow in 3D

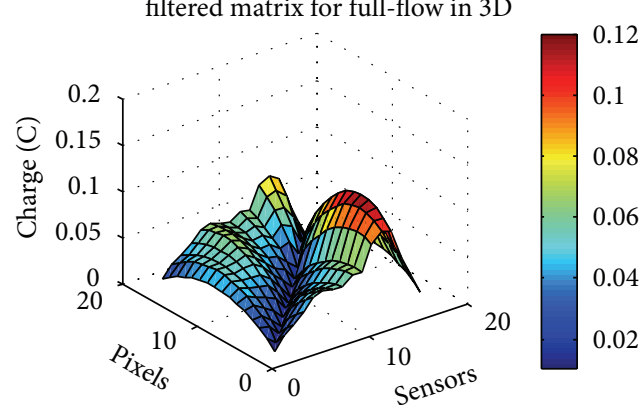

(d2)

FIGURE 12: The concentration profile using the square matrix in 2D and 3D for the four flow regimes using PRDM. 
PRD conc. profile with mesh elements coord. in 3D for 1/4-flow

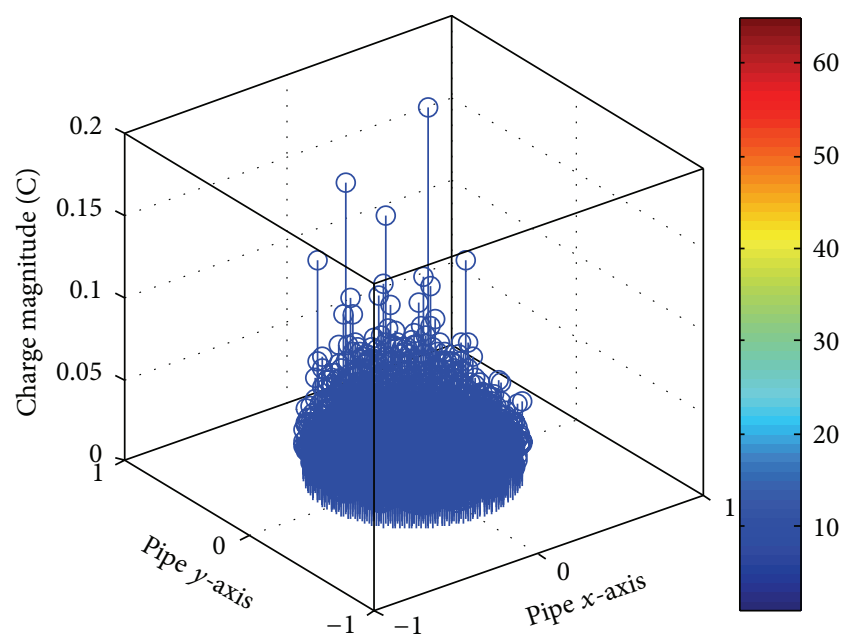

(a)

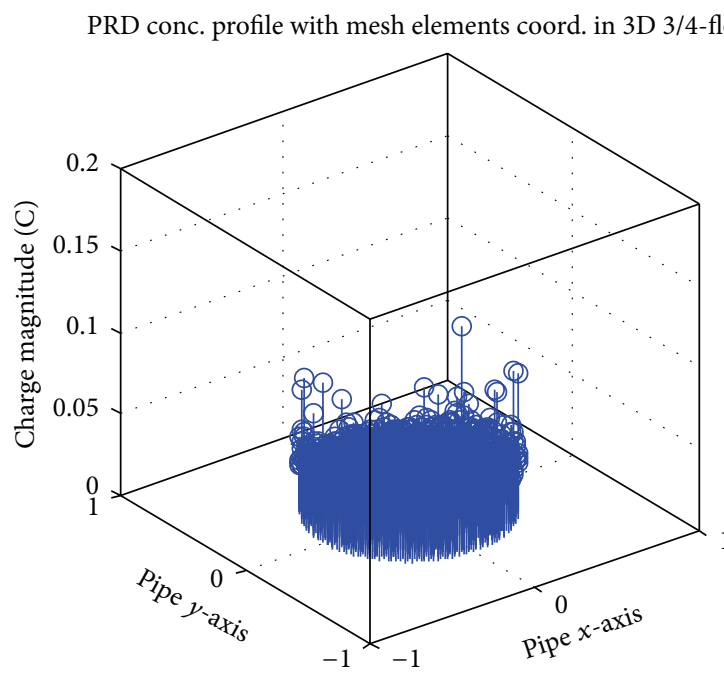

(c)
PRD conc. profile with mesh elements coord. in 3D 1/2-flow

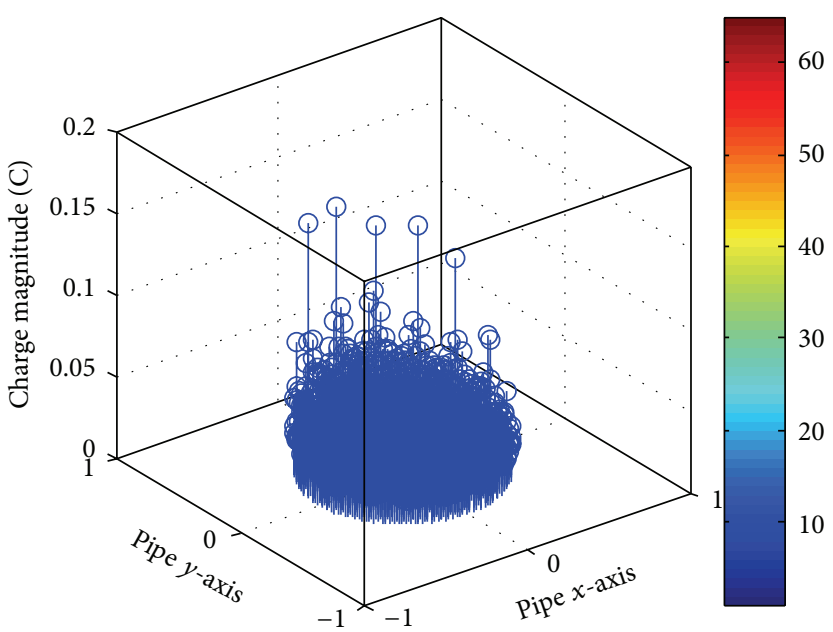

(b)

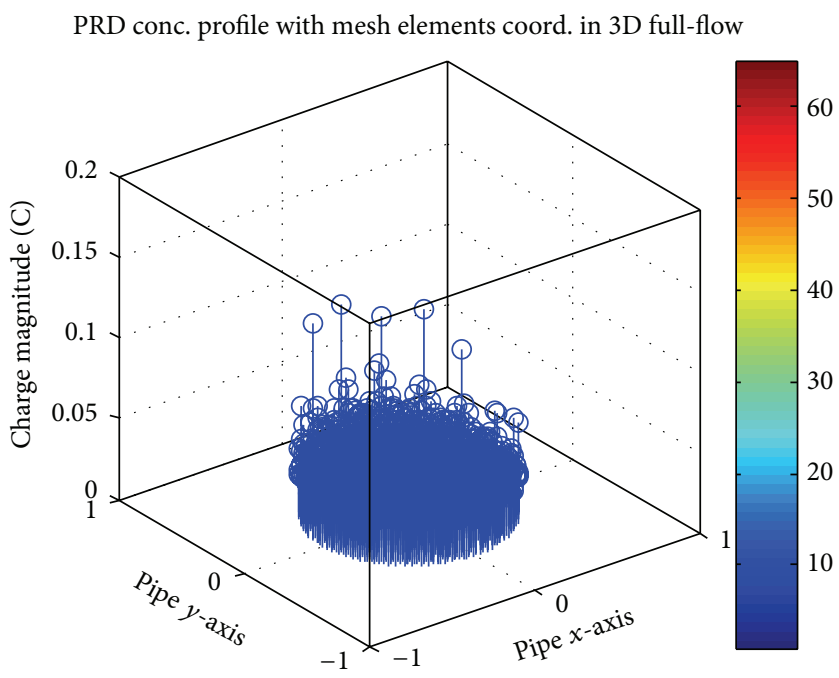

(d)

FIGURE 13: The concentration profile in 3D in terms of mesh elements using the PRDM: (a) 1/4-flow, (b) 1/2-flow, (c) 3/4-flow, and (d) full-flow.

TABLE 6: Extract from the pro rata matrix for full-flow regime.

\begin{tabular}{|c|c|c|c|c|c|}
\hline \multirow{2}{*}{ Elements $\left(e_{i}\right)$} & \multicolumn{5}{|c|}{ Sensors pro rata matrix $\left(S_{n}\right)$} \\
\hline & $S_{1}$ & $S_{2}$ & $S_{3}$ & - & $S_{16}$ \\
\hline$e_{1}$ & $1.20 E-04$ & $2.01 E-04$ & $1.73 E-04$ & - & $1.17 E-04$ \\
\hline$e_{2}$ & $1.12 E-04$ & $1.48 E-04$ & $9.78 E-05$ & - & $1.37 E-04$ \\
\hline$e_{3}$ & $1.21 E-04$ & $2.06 E-04$ & $1.80 E-04$ & - & $1.16 E-04$ \\
\hline$e_{4}$ & $1.12 E-04$ & $1.50 E-04$ & $1.04 E-04$ & - & $1.34 E-04$ \\
\hline - & - & - & - & - & - \\
\hline$e_{1371}$ & $2.45 E-05$ & $2.93 E-06$ & $3.63 E-05$ & - & $5.60 E-05$ \\
\hline
\end{tabular}

across the pipeline. This inhomogeneous distribution of the particles can be observed in the photographs taken using a digital camera, as shown in Figure 14. The figures correspond to the reconstructed particle distribution pattern across the pipe cross-section.
5.3. The Results of the Stability Analysis. The condition number is one of the measures of the numerical stability of a matrix. In the determination of the stability, if the condition number is finite and not more than $10^{3}$, the system is stable. Won et al. [29] stated that if condition number is more than 


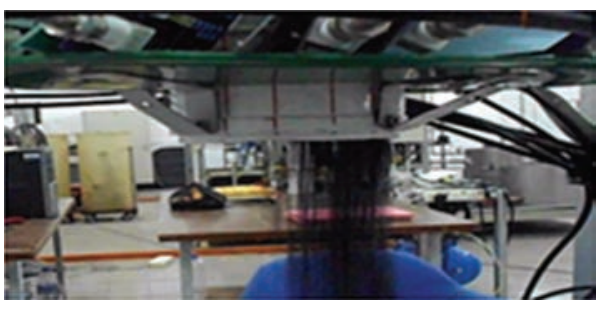

(a)

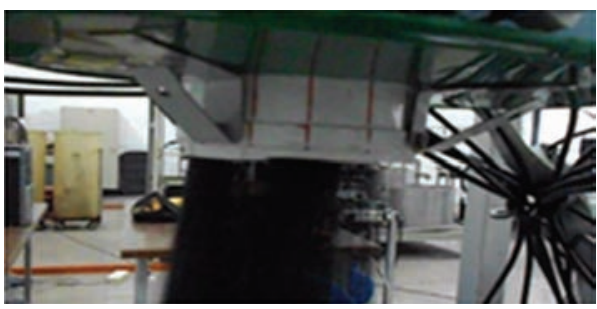

(c)

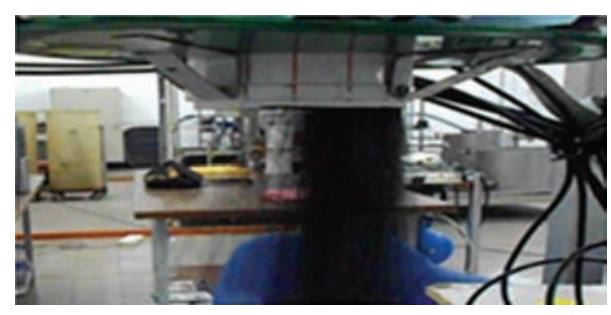

(b)

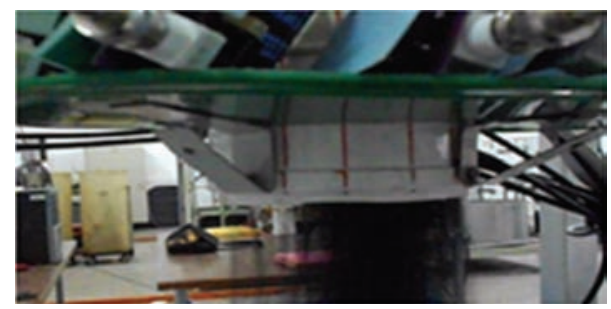

(d)

FIGURE 14: The photographs captured during experimentation: (a) quarter-flow, (b) half-flow, (c) three-quarter-flow, and (d) full-flow.

TABLE 7: The real induced DC voltage captured by each of the installed sensors due to charges on the flowing particles used for the concentration profiling.

\begin{tabular}{|c|c|c|c|c|c|c|c|c|c|c|c|c|c|c|c|c|}
\hline \multirow{2}{*}{ Flow regimes } & \multicolumn{16}{|c|}{ Voltages $\left(V_{s}\right)$ captured by sensors } \\
\hline & $S_{1}$ & $S_{2}$ & $S_{3}$ & $S_{4}$ & $S_{5}$ & $S_{6}$ & $S_{7}$ & $S_{8}$ & $S_{9}$ & $S_{10}$ & $S_{11}$ & $S_{12}$ & $S_{13}$ & $S_{14}$ & $S_{15}$ & $S_{16}$ \\
\hline 1/4-flow & 0.138 & 0.238 & 0.147 & 0.071 & 0.079 & 0.111 & 0.119 & 0.041 & 0.178 & 0.144 & 0.587 & 0.930 & 0.900 & 1.090 & 0.548 & 0.190 \\
\hline 1/2-flow & 0.366 & 0.253 & 0.333 & 0.174 & 0.202 & 0.274 & 0.233 & 0.237 & 0.370 & 0.951 & 1.514 & 1.714 & 1.820 & 1.312 & 1.196 & 0.937 \\
\hline 3/4-flow & 0.939 & 1.036 & 1.000 & 0.903 & 1.179 & 1.001 & 1.076 & 1.108 & 1.270 & 1.007 & 0.395 & 0.494 & 0.411 & 0.423 & 1.125 & 0.824 \\
\hline Full-flow & 0.539 & 0.660 & 0.660 & 0.717 & 0.811 & 0.796 & 0.732 & 0.700 & 0.797 & 0.800 & 1.166 & 1.383 & 1.465 & 1.116 & 0.880 & 0.596 \\
\hline
\end{tabular}

TABLE 8: Condition numbers of the sensitivity $(S)$ matrix used.

\begin{tabular}{lcc}
\hline$S / N$ & Matrix type & Condition number \\
\hline 1 & Raw rectangular matrix generated from the system equation of the & 4 \\
2 & size $[1371 \times 16]$ & 16 \\
3 & The compressed $[16 \times 16]$ square raw matrix & 400 \\
4 & The filtered raw rectangular matrix $[1371 \times 16]$ & 91 \\
\hline
\end{tabular}

$10^{3}$, the system is ill-conditioned and unstable. The ideal condition number is 1 for an identity matrix as defined by Strang [30]. However, the MATLAB package was used to carry out the analysis of the condition number of the matrices used for the reconstruction of the concentration profile or the tomography images of the flowing solid particles through a pipeline. The required basic matrix used is the sensitivity matrix. In this work, four sensitivity matrices were developed and used in the image reconstruction and the condition numbers are presented in Table 8.

From Table 8, it can be seen that four condition numbers were computed. The first matrix was obtained from the system equation that described the distribution of the electric charge carried by the flowing solid particles across the conveying pipeline cross-section. The charge is distributed in each of the 1371 mesh elements as sensed by the 16 installed electrodynamic sensors that detect the charges on the flowing solid particles. This formed the matrix of size $[1371 \times 16]$ that gave the condition number of 4 in row 1 in Table 8 . To apply the LBP and FBP image reconstruction methods, the rectangular matrix is compressed to a matrix of size $[16 \times 16]$ through the transposition method that gave the condition number of 16 as shown in Table 8 in row 2.

To improve the qualities of the image, matrix filtering as suggested by Rahmat [31] was employed in the original matrix and then compressed which gave the condition numbers as 400 and 91 in rows 3 and 4, respectively. Table 8 shows some important phenomenon that can be observed, such as the effect of matrix filtering that increased the condition number. This implies that filtering of matrix decreases the stability of the system. The other phenomenon is that matrix compression also increases the condition number but filtering is higher. To reconstruct the concentration profiles for the four flow regimes, other matrices were developed using 
TABle 9: Condition numbers of the image matrix.

\begin{tabular}{llcc}
\hline$S / N$ & Matrix type & Condition number \\
\hline 1 & 1/4-flow unfiltered real image matrix used in PRD only (rectangular matrix) & 45 \\
2 & 1/2-flow unfiltered real image matrix used in PRD only (rectangular matrix) & 22 \\
3 & 3/4-flow unfiltered real image matrix used in PRD only (rectangular matrix) & 6.9 & $2.0 E+3$ \\
4 & Full-flow unfiltered real image matrix used in PRD (rectangular matrix) & 480 \\
5 & $1 / 4$-flow compressed unfiltered real image matrix used in LBP, FBP, and PRD (square matrix) & 79 \\
6 & $1 / 2$-flow compressed unfiltered real image matrix used in LBP, FBP, and PRD (square matrix) & 40 \\
7 & 3/4-flow compressed unfiltered real image matrix used in LBP, FBP, and PRD (square matrix) & $2.7 E+03$ \\
8 & Full-flow compressed unfiltered real image matrix used in LBP, FBP, and PRD (square matrix) & $1.7 E+03$ \\
9 & 1/4-flow filtered real image matrix used in PRD only (rectangular matrix) & 740 \\
10 & 1/2-flow filtered real image matrix used in PRD only (rectangular matrix) & 540 \\
11 & 3/4-flow filtered real image matrix used in PRD only (rectangular matrix) & 690 \\
12 & Full-flow filtered real image matrix used in PRD only (rectangular matrix) & 400 & 190 \\
13 & 1/4-flow compressed filtered real image matrix used in LBP, FBP, and PRD (square matrix) & 130 \\
14 & 1/2-flow compressed filtered real image matrix used in LBP, FBP, and PRD (square matrix) & \\
15 & 3/4-flow compressed filtered real image matrix used in LBP, FBP, and PRD (square matrix) & \\
16 & Full-flow compressed filtered real image matrix used in LBP, FBP, and PRD (square matrix) & \\
\hline
\end{tabular}

the data captured by the sensors and the system's sensitivity matrices of Table 8 for different flow regimes. These sets of new matrices are called the image matrices. The condition numbers of the image matrices developed for PRD, LBP, and FBP using the FEM data obtained from the model equation are shown in Table 9. In this paper, only PRD method was used for the concentration profile.

In Table 9, the image matrices were obtained by back projecting and pro rata distributing of the voltages captured by the 16 installed electrodynamic sensors, onto the compressed unfiltered sensitivity and the compressed filtered matrices to give the image matrices. The captured data are for each of the 4 flow regimes. It can be observed that some of the image matrices are applicable to PRD while not all are applied to LBP and FBP, which is because PRD method can be applied to any matrix shape. It is worth noting that the condition numbers increase with increase in the baffle area in each of the categories, which means that condition number is more in 1/4-flow than the full-flow. This is because the difference between the maximum and minimum value of the image matrix increases with reduction in the flow channel, as can be seen in the bar chart of Figure 10.

Going by the standard that any matrix of a system whose condition number is not more than $10^{3}$ is a well conditioned and stable system [32], then most of the condition numbers in Tables 8 and 9 are well within the stable condition. Comparing the condition numbers obtained in the previous research on electric charge tomography reported by Isa [16], the condition numbers obtained using the proposed FEM system are the least so far. This is because Isa [16] reported that singular value decomposition (SVD) gave a condition number of $1.4 E+23$ which is highly unstable, while Rahmat et al. [25] were able to obtain the highest value of $3.1 E+18$ and the least value of 130 . With the new FEM approach proposed in this paper, the condition numbers presented in Tables 8 and 9 show a significant breakthrough in terms of the stability of the propose method, with the highest condition number of $2.7 E+03$ and the least of 4.0 .

\section{Discussion}

In the finite element analysis, mesh elements should completely cover the problem domain; triangular elements should be equilateral while quadrilateral shaped elements should be squared. In line with these requirements, 1371 triangular elements were structurally generated in the sensing zone as can be seen in Figure 5 using the developed MATLAB codes. Particles move as a stream through pipeline where the charges carried by the moving particles are captured by electrodynamic sensors installed around the sensing zone.

The captured data are the voltage outputs from each of the sensors. Experiments were conducted and the average output voltages of each sensor under different flow regimes are shown in Table 7 and presented in bar chart of Figure 10, which proved that electrodynamic sensor is ideal and sensitive for the measurement of charges carried out by particles. The pro rata distribution procedure was carried on the captured voltage to generate the image data used for the reconstruction of the concentration profiles presented in Figures 11, 12, and 13 for the four different flow regimes. It is interesting to note that the patterns for fullflow and three-quarter-flow as well as half-flow and quarterflow are similar, with differences in the charge magnitudes (as can be read from the grey scale). This is because of the nature of measurement instruments, which does not give homogeneous particle distribution in the cross-section of the pipeline and these are practically shown in the experimental photographs of Figure 14. Another factor is that when the particles hit the baffles, the particle scatters due to gravitational force and induces charges to the other sensors, and this was observed during the experimentation process and can be seen in the captured voltage pattern in the bar chart of Figure 10. 
Condition number analysis was carried out to determine the stability of the system, and the results show that the system is well conditioned and stable as explained in Section 5.

\section{Conclusions}

The process tomography imaging is an act of obtaining the cross-sectional image of distribution of material in a region of interest, such as pipeline and process vessels usually applied in industrial processes. In this paper, FEM was used to generate mesh elements (which serves as the image pixels) across a pipe cross-section and the central coordinates of each of the mesh elements were calculated with which the system equation was developed. The system equation was used to compute the system's sensitivity matrix. The computed sensitivity matrix was used to compute the image matrices by the use of voltage data, measured from the 16 electrodynamic sensors. For the experimentation, four artificial flow regimes or profiles were created and the electrodynamic sensors were used to capture voltage data via Keithley Instruments. The captured voltage data were used for the reconstruction of the concentration profiles of the solid particles using the PRD technique. Stability analysis was carried out from which the results show that the approach gave the most stable data in process tomography system so far. The newly introduced PRD technique gave a good representation of the concentration profile of the flowing particles in both the square and triangular matrix. MATLAB codes were developed and used for the realization of the concentration profile reconstruction otherwise called the tomography image.

\section{Conflict of Interests}

The authors declare that there is no conflict of interests regarding the publication of this paper.

\section{References}

[1] C. Xu, J. Li, H. Gao, and S. Wang, "Investigations into sensing characteristics of electrostatic sensor arrays through computational modelling and practical experimentation," Journal of Electrostatics, vol. 70, no. 1, pp. 60-71, 2012.

[2] T. York, H. McCann, and K. B. Ozanyan, "Agile sensing systems for tomography," IEEE Sensors Journal, vol. 11, no. 12, pp. 30863105, 2011.

[3] M. Machida and B. Scarlett, "Process tomography system by electrostatic charge carried by particles," IEEE Sensors Journal, vol. 5, no. 2, pp. 251-259, 2005.

[4] L. Peng, Y. Zhang, and Y. Yan, "Characterization of electrostatic sensors for flow measurement of particulate solids in squareshaped pneumatic conveying pipelines," Sensors and Actuators $A$, vol. 141, no. 1, pp. 59-67, 2008.

[5] R. G. Green and R. Thorn, "Sensor systems for lightly loaded pneumatic conveyors," Powder Technology, vol. 95, no. 1, pp. 7992, 1998.

[6] Y. Yan, "Mass flow measurement of bulk solids in pneumatic pipelines," Measurement Science and Technology, vol. 7, no. 12, pp. 1687-1706, 1996.
[7] Y. Yan, B. Byrne, S. Woodhead, and J. Coulthard, "Velocity measurement of pneumatically conveyed solids using electrodynamic sensors," Measurement Science and Technology, vol. 6, no. 5, article no. 013, pp. 515-537, 1995.

[8] M. F. Rahmat, M. D. Isa, K. Jusoff, T. A. Hussin, and S. M. Rozali, "Image reconstruction algorithm for electrical charge tomography system," American Journal of Applied Sciences, vol. 7, no. 9, pp. 1254-1263, 2010.

[9] B. Zhou, J. Zhang, C. Xu, and S. Wang, "Image reconstruction in electrostatic tomography using a priori knowledge from ECT," Nuclear Engineering and Design, vol. 241, no. 6, pp. 1952-1958, 2011.

[10] M. F. Rahmat, R. Abdul Rahim, and H. A. Sabit, "Application of neural network and electrodynamic sensor as flow pattern identifier," Sensor Review, vol. 30, no. 2, pp. 137-141, 2010.

[11] M. F. Rahmat and N. S. Kamaruddin, "Application of fuzzy logic and electrodynamic sensors as flow pattern identifier," Sensor Review, vol. 32, no. 2, pp. 123-133, 2012.

[12] M. F. Rahmat and I. T. Thuku, "Review of tomographic imaging using finite element method," Sensors and Transducers, vol. 135, no. 12, pp. 1-19, 2011.

[13] I. T. Thuku, M. F. Rahmat, T. Tajdari, N. Abdul Wahab, and A. A. Yusuf, "2-D finite-element modeling of electrostatic sensor for tomography system," Emerald: Sensor Review, vol. 33, pp. 104113, 2013.

[14] S. M. Bragg, Financial Analysis: A Controller's Guide, John Wiley \& Sons, Hoboken, NJ, USA, 2nd edition, 2007.

[15] F. Jonathan and L. Jeremy, Pro-Rata Matching in One-Tick Markets, EC3R 6DU, London, UK, 2012.

[16] M. D. Isa, Least square with regularization algorithm for electric charge tomography system [Ph.D. thesis], Control and Mechatronics Engineering, Universiti Teknologi Malaysia, Johor Bahru, Malaysia, 2011.

[17] R. A. Williams, C. G. Xie, F. J. Dickin, S. J. R. Simons, and M. S. Beck, "Multi-phase flow measurements in powder processing," Powder Technology, vol. 66, no. 3, pp. 203-224, 1991.

[18] H. J. Stanley, Finite-Element Methods in Electromagnetics, CRC Press, Albuquerque, NM, USA, 2010.

[19] Y. W. Kwon and H. Bang, The Finite Element Method Using MATLAB, CRC Mechanical Engineering Series, Chapman \& Hall/CRC, Boca Raton, Fla, USA, 2nd edition, 2000.

[20] R. G. Green, M. F. Rahmat, K. Evans, A. Goude, M. Henry, and J. A. R. Stone, "Concentration profiles of dry powders in a gravity conveyor using an electrodynamic tomography system," Measurement Science and Technology, vol. 8, no. 2, pp. 192-197, 1997.

[21] F. Daniel, A Student Guide to Maxwell's Equations, Cambridge University Press, New York, NY, USA, 2008.

[22] B. L. Theraja and A. K. Theraja, A Textbook of Electrical Technology, S. Chand \& Company, New Delhi, India, 24th edition, 2005.

[23] M. Machida and M. Kaminoyama, "Sensor design for development of tribo-electric tomography system with increased number of sensors," Journal of Visualization, vol. 11, no. 4, pp. 375-385, 2008.

[24] A. C. Polycarpou, "Introduction to the finite element method in electromagnetics," Synthesis Lectures on Computational Electromagnetics, vol. 4, pp. 1-126, 2006.

[25] M. F. Rahmat, M. D. Isa, R. A. Rahim, and T. A. R. Hussin, "Electrodynamics sensor for the image reconstruction process in an electrical charge tomography system," Sensors, vol. 9, no. 12, pp. 10291-10308, 2009. 
[26] H. Yan, C. Liu, and J. Gao, "Electrical capacitance tomography image reconstruction based on singular value decomposition," in Proceedings of the 5th World Congress on Intelligent Control and Automation (WCICA '04), vol. 4, pp. 3783-3786, Hangzhou, China, June 2004.

[27] X. Chen and K. K. Phoon, "Applications of symmetric and nonsymmetric MSSOR preconditioners to large-scale Biot's consolidation problems with nonassociated plasticity," Journal of Applied Mathematics, vol. 2012, Article ID 352081, 15 pages, 2012.

[28] W. Q. Yang, D. M. Spink, T. A. York, and H. McCann, “An image-reconstruction algorithm based on Landweber's iteration method for electrical-capacitance tomography," Measurement Science and Technology, vol. 10, no. 11, pp. 1065-1069, 1999.

[29] J.-H. Won, J. Lim, S.-J. Kim, and B. Rajaratnam, "Conditionnumber-regularized covariance estimation," Journal of the Royal Statistical Society. Series B, vol. 75, no. 3, pp. 427-450, 2013.

[30] G. Strang, Introduction to Linear Algebra, Wellesley-Cambridge Press, Cambridge, Mass, USA, 4th edition, 2009.

[31] M. F. Rahmat, Instrumentation of particle conveying using electrical charge tomography [Ph.D. thesis], Control Engineering, Sheffield Hallam University, Sheffield, UK, 1996.

[32] F. Milinazzo, C. Zala, and I. Barrodale, "On the rate of growth of condition numbers for convolution matrices," IEEE Transactions on Acoustics, Speech, and Signal Processing, vol. 35, no. 4, pp. 471-475, 1987. 


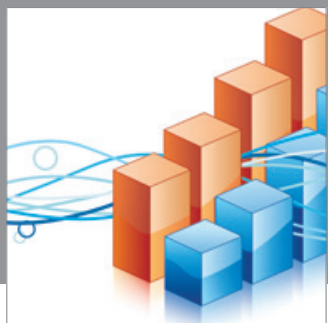

Advances in

Operations Research

mansans

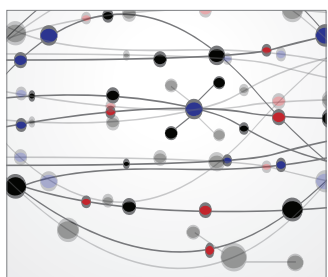

The Scientific World Journal
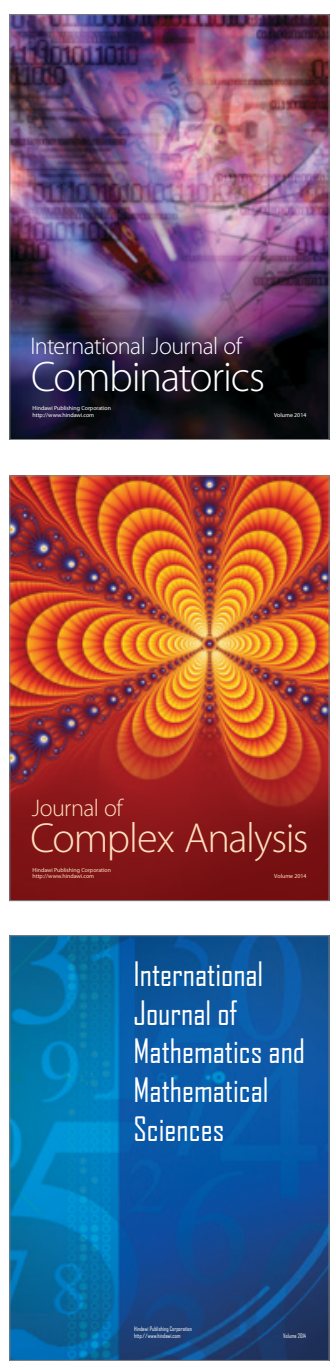
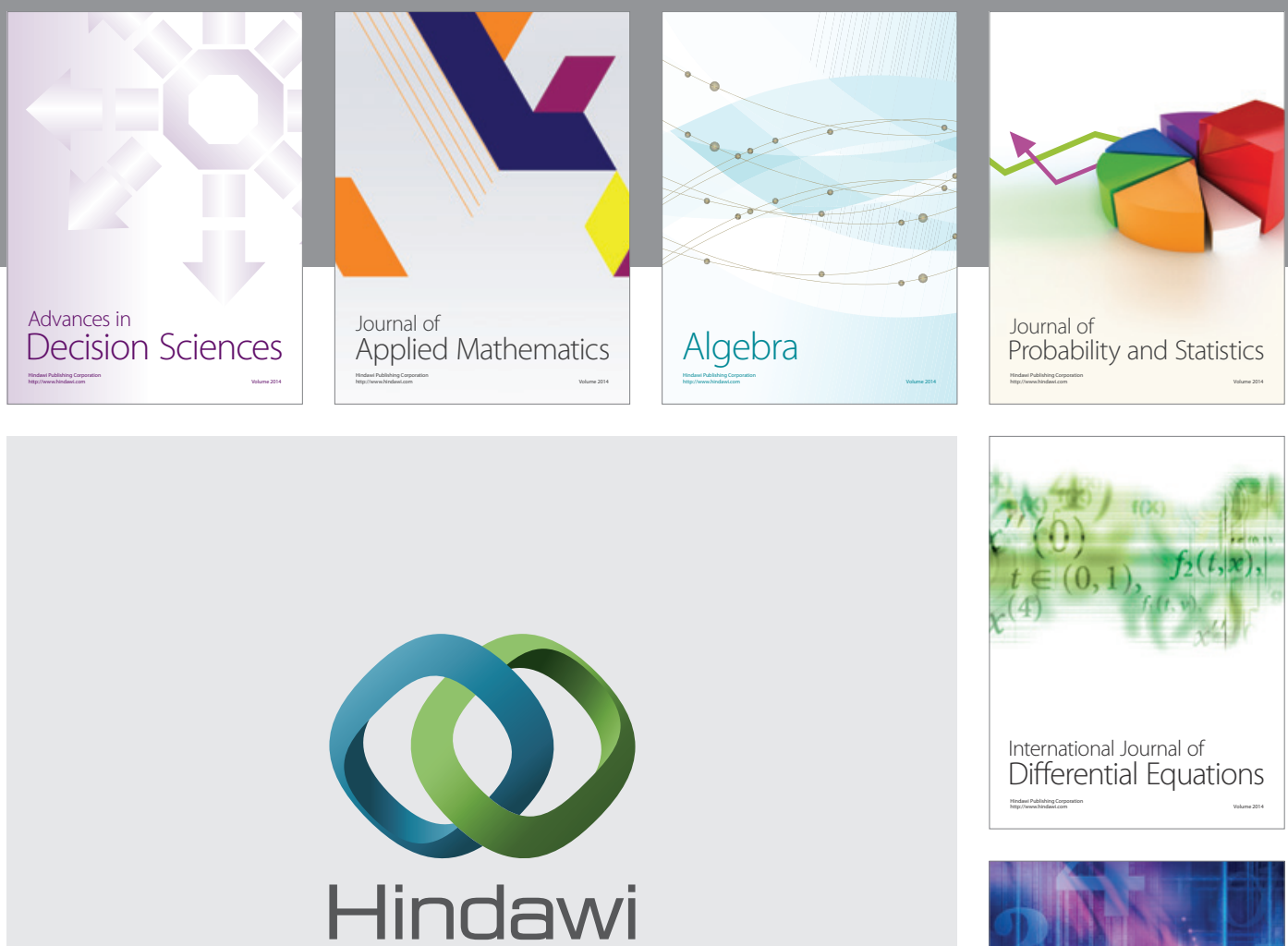

Submit your manuscripts at http://www.hindawi.com
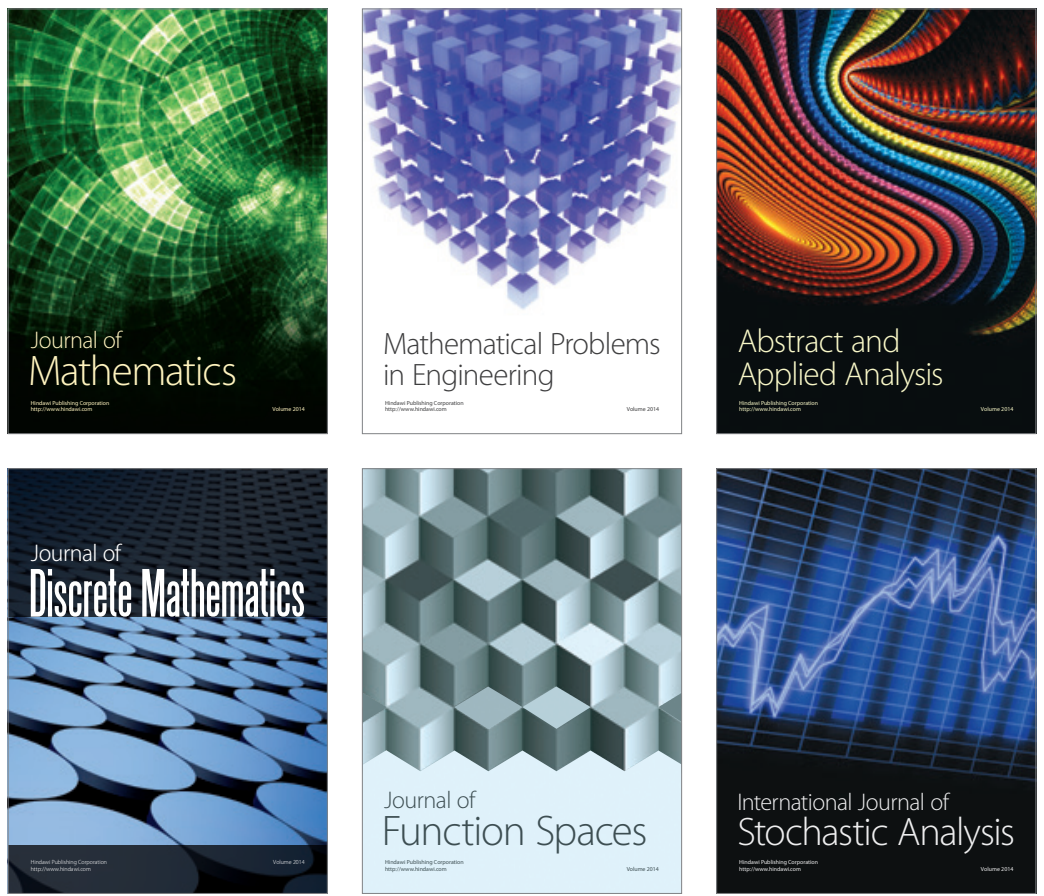

Journal of

Function Spaces

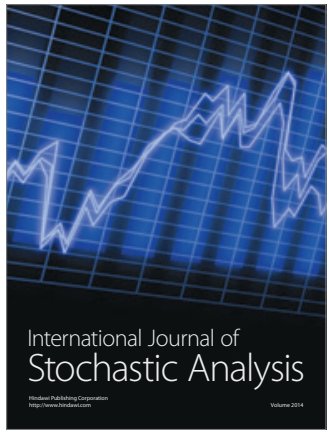

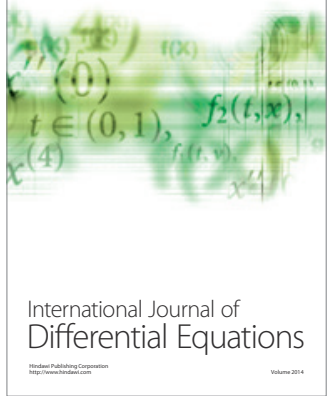
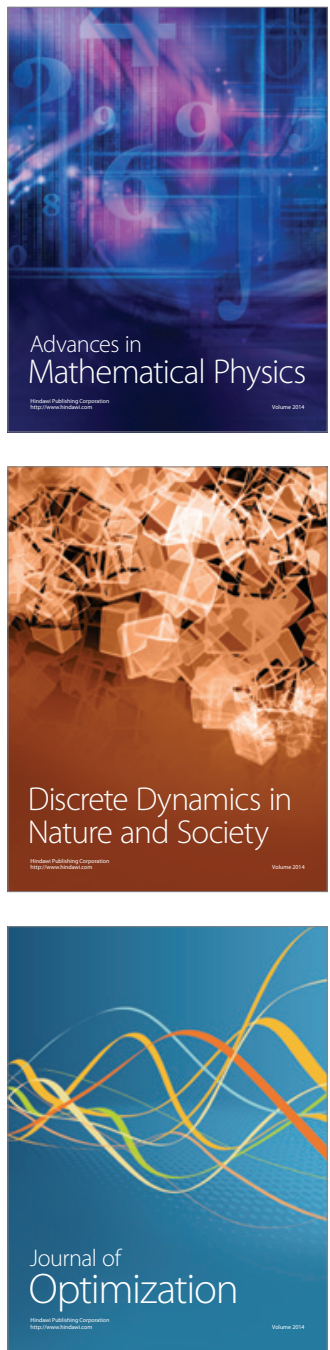